\title{
New Liquid Holdup Models in both Preloading \& Flooding Region and Flood Velocity Model to Identify Flooding for Sheet Metal Structured Packings
}

\author{
Anil Krishna Jammula a *, James Rob Whiteley a , Michael R. Resetarits ${ }^{\text {a }}$, Tony J. Cai ${ }^{\text {b }}$ \\ a: Oklahoma State University, 202 Whitehurst, Stillwater, OK, USA - 74078 \\ b: Fractionation Research Inc., 424 Squires Landing BIvd, Stillwater, OK, USA - 74074 \\ *: Corresponding author. Email: anil.jammula@okstate.edu
}

Keywords: Liquid Holdup, preloading region, structured packing, no packing specific constants 


\section{Structured packing dimensions}

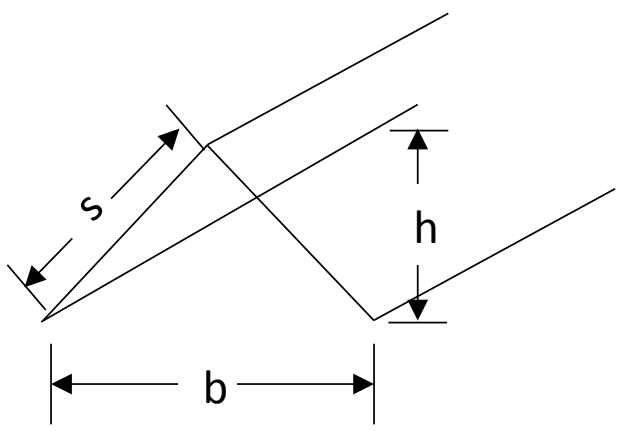

Fig. S1. Packing crimps and illustration of the geometric variables associated with such crimps

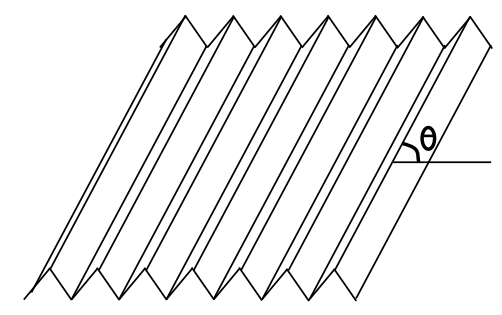

Fig. S2. Packing corrugation angle with respect to horizontal axis 


\section{Example Problem 1:}

1. An air/water column is being operated at atmospheric conditions using Mellapak 250Y. Please calculate the preloading region liquid holdup when the column has a constant liquid flow rate of $32 \mathrm{~m}^{3} / \mathrm{m}^{2} \mathrm{~h}$ and a vapor flow rate of $1.95 \mathrm{~m} / \mathrm{s}$. Please use the conditions below for the calculations:

$\begin{array}{lll}\text { Water Density } & = & 1000 \mathrm{~kg} / \mathrm{m}^{3} \\ \text { Water Viscosity } & = & 10 \mathrm{cp} \\ \text { Air Density } & = & 1.3 \mathrm{~kg} / \mathrm{m}^{3} \\ \text { Packing Area (a) } & = & 250 \mathrm{~m}^{2} / \mathrm{m}^{3} \\ \text { Void fraction ( } \varepsilon \text { ) } & = & 97.5 \mathrm{~m}^{3} / \mathrm{m}^{3} \\ \text { Corrugation angle } & = & 45\end{array}$

Answer: $\quad$ Given, Superficial Liquid velocity $\mathrm{u}_{\mathrm{L}}=0.0089 \mathrm{~m} / \mathrm{s}$

- $\quad$ At $\mathrm{u}_{\mathrm{G}}=1.95 \mathrm{~m} / \mathrm{s}$

From eqn. 6,

$$
\begin{gathered}
R e=\frac{1000 \frac{\mathrm{kg}}{\mathrm{m}^{3}} \times 0.0089 \frac{\mathrm{m}}{\mathrm{s}}}{250 \frac{\mathrm{m}^{2}}{\mathrm{~m}^{3}} \times\left(\frac{10}{10000}\right) P a \cdot s}=35.6 \\
h_{L, \text { preload }}=0.114 \frac{250^{1.23} 35.6^{0.41}}{(1-(97.5 / 100))^{0.28}}\left(\frac{\left(\frac{10}{10000}\right)^{2}}{1000^{2} \times 9.81 \times \sin 45}\right)^{1 / 3}
\end{gathered}
$$




$$
=0.0647 \mathrm{~m}^{3} / \mathrm{m}^{3}
$$

Actual experimental liquid holdup $=0.0642 \mathrm{~m}^{3} / \mathrm{m}^{3}$

$$
\text { Error, } \%=a b s\left(\frac{0.0647-0.0642}{0.0642}\right) \times 100=0.7 \%
$$

Note: In OkState preload model, if the exponent of the viscosity term $\left[\left(\frac{\mu_{L}^{2}}{\rho^{2} g \sin \theta}\right)^{\frac{1}{3}}\right]$ is approximated as 0.33 instead of (1/3), the model's accuracy is lessened. 


\section{Appendix 1: Preloading Region Literature Models performance using experimental}

\section{liquid holdup data (Table 1)}

\section{Alix-1 Model 1:}

$$
\begin{gathered}
h_{L}=h_{L_{0}}+K^{P} \frac{\Gamma}{\rho_{L}}\left(\frac{\mu_{L}}{\mu_{w}}\right)^{\frac{1}{3}} \\
\Gamma=\rho_{L} \frac{V_{S L}}{a_{g}} \\
K^{P}=691\left[\mathrm{~s} \mathrm{~m}^{-2}\right] \\
h_{L_{0}}=6.3 \%
\end{gathered}
$$

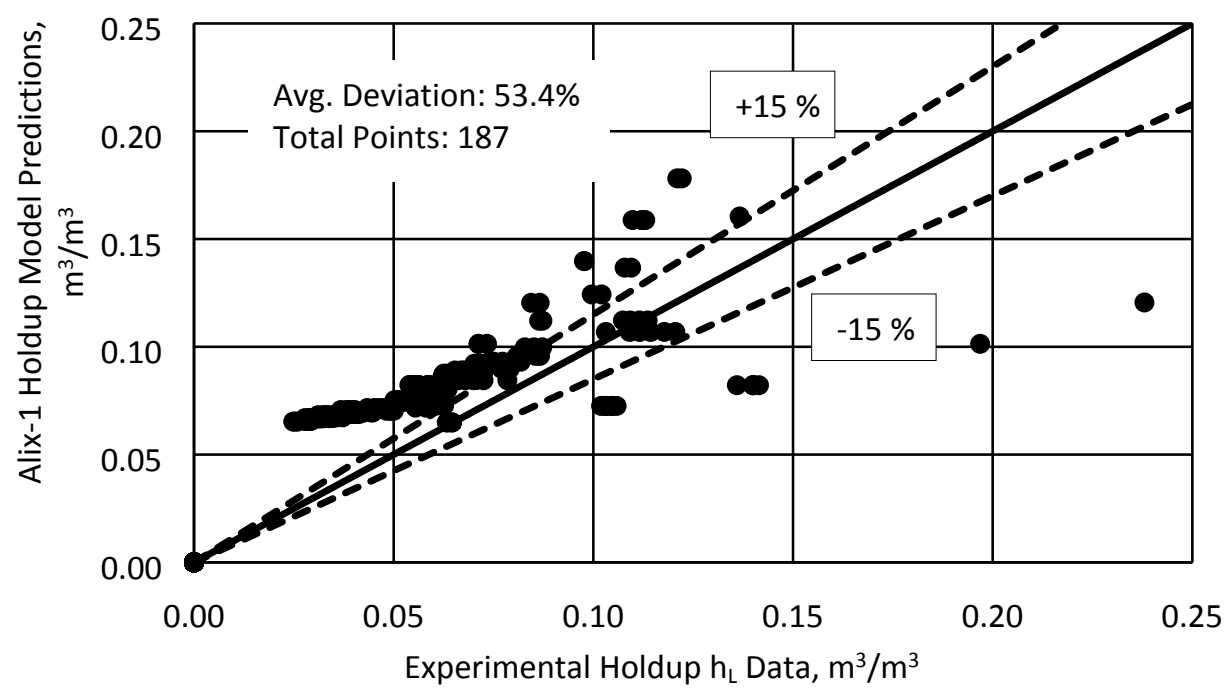

Fig. S3. Alix-1 preloading region model predictions vs experimental liquid holdup data 


\section{Alix-2 Model ${ }^{1}$ :}

$$
\begin{gathered}
h_{L}=h_{L_{0}}+C_{1} \Gamma^{0.4}\left(\frac{\mu_{L}}{\mu_{w}}\right)^{\frac{1}{3}} \\
R e_{L}=\frac{4 \Gamma}{\mu_{L}}=\frac{4 \rho_{L} V_{S L}}{a_{g} \mu_{L}} \\
C_{1}=0.2683\left[\mathrm{~s} \mathrm{~m}^{-2}\right] \\
R e_{L_{0}}=\frac{4 \Gamma_{0}}{\mu_{L}}=800 \\
h_{L_{0}}=0.032 \frac{\Gamma}{\Gamma_{0}} \text { for } \operatorname{Re}_{L} \leq R e_{L_{0}} \\
h_{L_{0}}=0.032 \text { for } \operatorname{Re}_{L}>\operatorname{Re}_{L_{0}}
\end{gathered}
$$

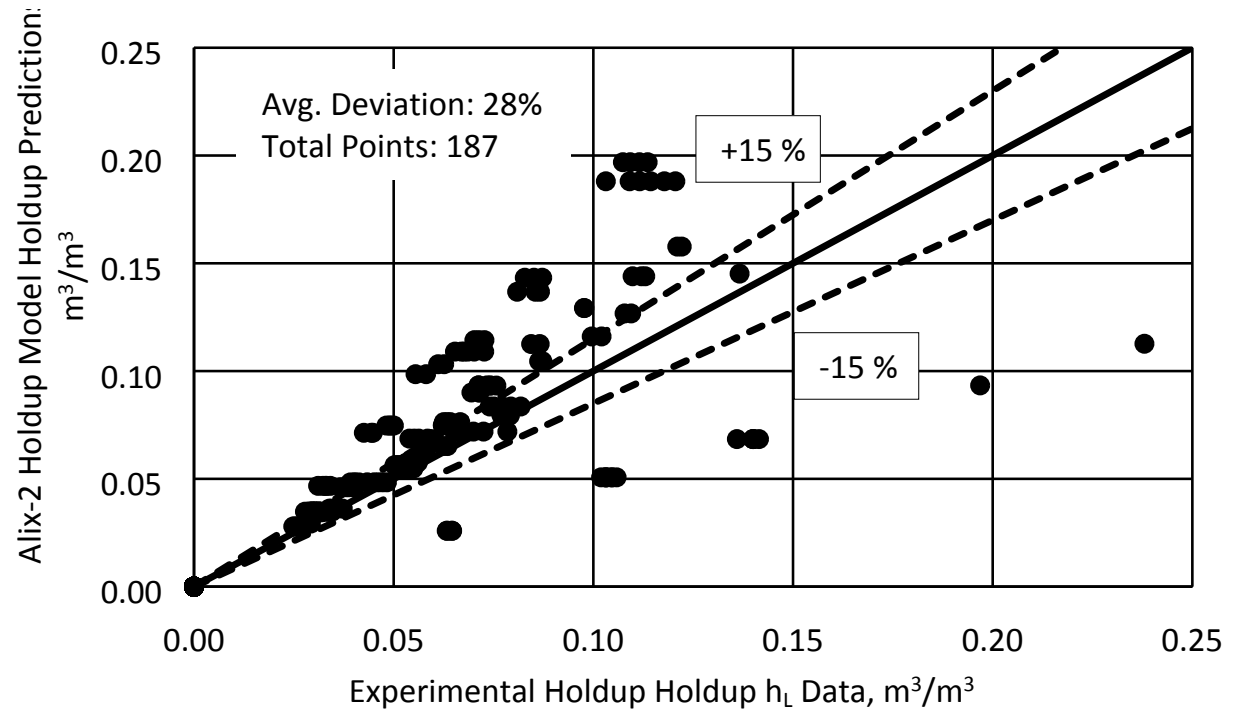

Fig. S4. Alix-2 preloading region model predictions vs experimental liquid holdup data 


\section{Delft Model ${ }^{2-5}$ :}

$$
\begin{gathered}
\alpha_{L}=\arctan \left[\frac{\cos (90-\alpha)}{\sin (90-\alpha) \cos \left[\arctan \left(\frac{b}{2 h}\right)\right]}\right] \\
\delta=\left(\frac{3 \mu_{L} u_{L s}}{\rho_{L} g a_{P} \sin \alpha_{L}}\right)^{\frac{1}{3}} \\
h_{L}=\delta a_{P}
\end{gathered}
$$

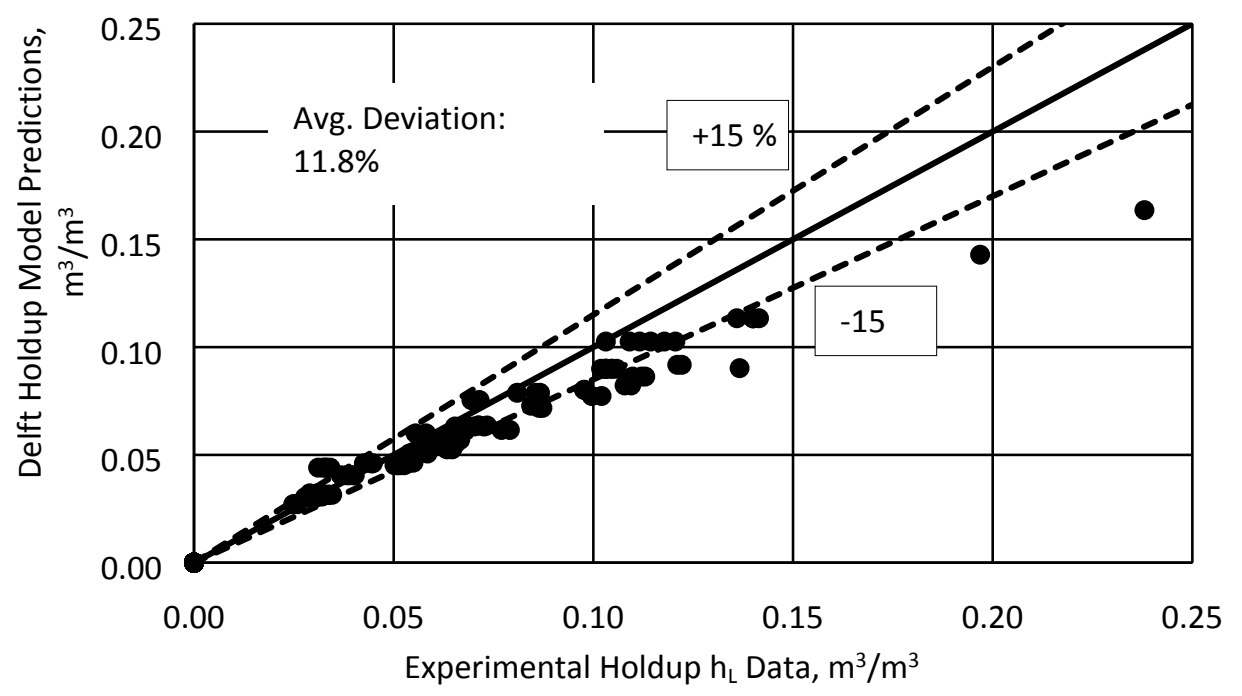

Fig. S5. Delft preloading region model predictions vs experimental liquid holdup data 


\section{Gualito Model ${ }^{6}$ :}

$$
\begin{gathered}
\left(\frac{\Delta P}{\Delta Z}\right)_{d r y}=\left(\frac{\rho_{G}}{\rho_{(\text {air }, 1 \text { bar })}}\right)^{0.4}\left(\frac{0.177 \rho_{G} U_{G S}^{2}}{S \epsilon^{2}(\sin \theta)^{2}}+\frac{88.77 u_{G} U_{G S}}{S^{2} \epsilon \sin \theta}\right) \\
F_{t}=\frac{a_{e}}{a_{p}}=\frac{\left(W e_{L} F r_{L}\right)^{0.15} 29.12 S^{0.36}}{R e_{L}^{0.2} \epsilon^{0.6}(1-0.93 \cos \gamma)(\sin \theta)^{0.3}} \\
\left(\frac{\Delta P}{\Delta Z}\right)_{\text {flood }}=1500+65000 U_{L S}
\end{gathered}
$$

Solve below equations,

$$
\begin{aligned}
& h_{L}=\left[\frac{4 F_{t}}{S}\right]^{\frac{2}{3}}\left(\frac{3 \mu_{L} U_{L s}}{\rho_{L} \in \sin \theta\left[\left(\frac{\rho_{L}-\rho_{G}}{\rho_{L}}\right)\left(1-\frac{\left(\frac{\Delta P}{\Delta Z}\right)_{\text {new }}}{\left.\left(\frac{\Delta P}{\Delta Z}\right)_{\text {flood }}\right)}\right)\right.}\right)^{\frac{1}{3}} \\
& \frac{\Delta P}{\Delta Z}=\frac{\left(\frac{\Delta P}{\Delta Z}\right)_{d r y}}{\left[1-(0.614+71.35 S) h_{L}\right]^{5}}
\end{aligned}
$$




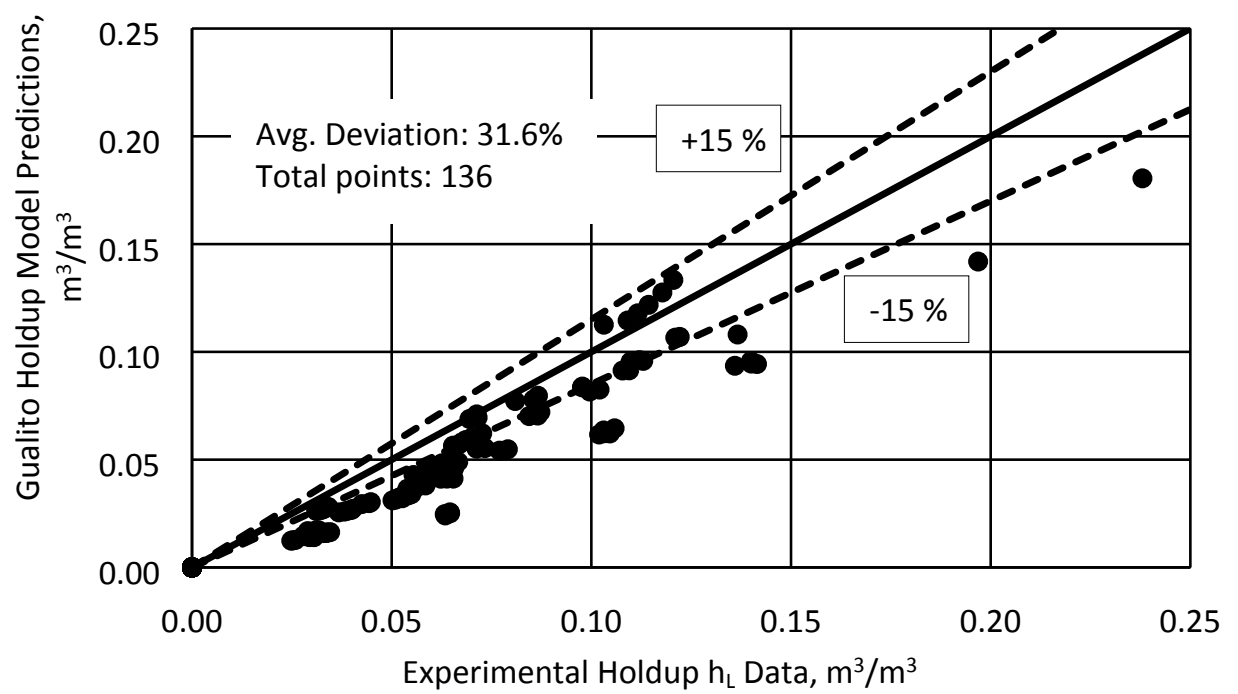

Fig. S6. Gualito preloading region model predictions vs experimental liquid holdup data

\section{Macias Model ${ }^{7}$ :}

$$
\begin{gathered}
d_{e q}=B h\left[\frac{1}{B+2 S}+\frac{1}{2 S}\right] \\
F i=\frac{F r}{R e}=\frac{\left(\frac{v_{s}^{2}}{g d_{e q}}\right)}{\frac{v_{s} d_{e q} \rho}{\eta}} \\
H_{t}=0.05557 F i^{0.3165}\left(\frac{3}{\sin ^{2} \varnothing}\right)^{0.8767}\left(d_{e q} a_{p}\right)^{2.405}
\end{gathered}
$$




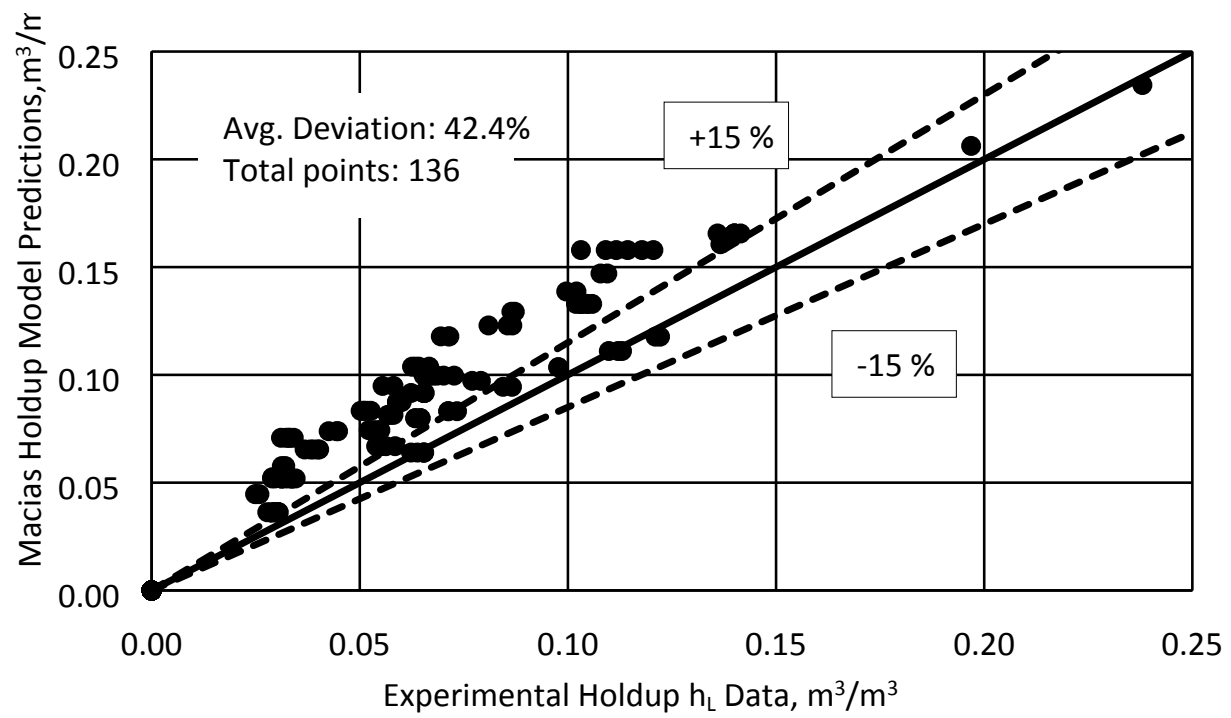

Fig. S7. Macias preloading region model predictions vs experimental liquid holdup data

6. Mackowiak-2 Model ${ }^{8}$ :

$$
h_{L}=0.465\left(\frac{u_{L}^{2} a}{g}\right)^{\frac{1}{3}}
$$




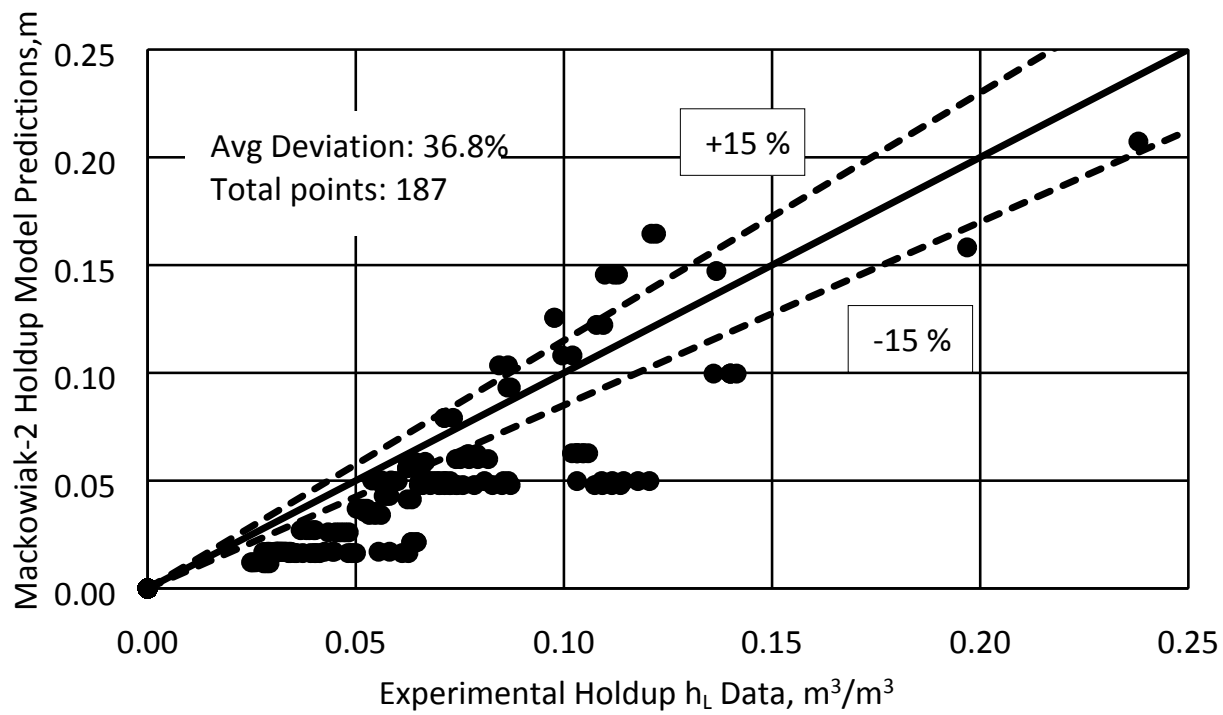

Fig. S8. Mackowiak-2 preloading region model predictions vs experimental liquid holdup data

7. Mackowiak Model ${ }^{9}$ :

$$
d_{P}=\frac{6(1-\varepsilon)}{a}
$$




$$
B_{L}=\left(\frac{\mu_{L}}{g^{2} \rho_{L}}\right)^{\frac{1}{3}}\left(\frac{u_{L}}{\varepsilon}\right)\left(\frac{1-\varepsilon}{\varepsilon d_{P}}\right)
$$

$$
h_{L}=2.2 B_{L}^{0.5}
$$

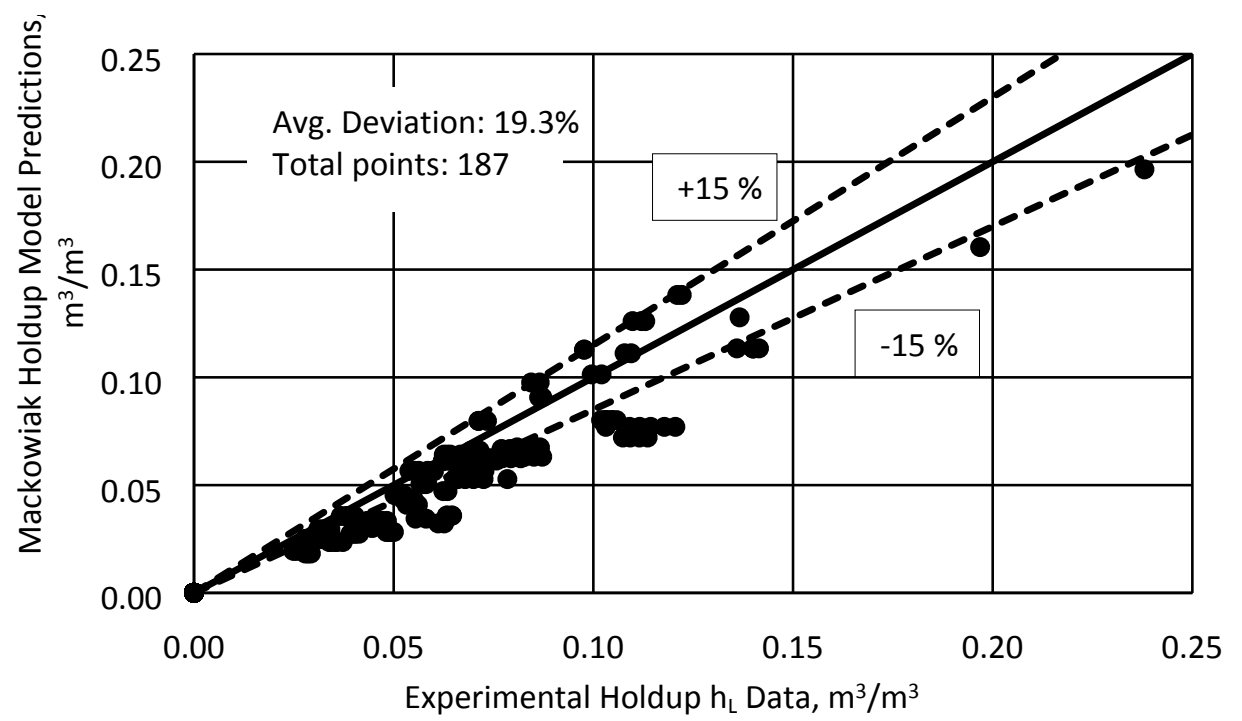

Fig. S9. Mackowiak-2 preloading region model predictions vs experimental liquid holdup data

\section{Pondebat Model ${ }^{10}$ :}




$$
\begin{gathered}
h_{L}=K_{3}\left[\frac{\mu_{L}}{\rho_{L} v_{\text {Water }}}\right]^{A_{5}} u_{L}^{A_{6}} \exp \left(K_{4} u_{V} \rho_{G}^{0.5}\right) \\
u_{L, E f f}=\left[\frac{3 u_{L}}{2 \text { Per }}\right]\left[\frac{(g \text { Per })}{3 v_{L} u_{L}}\right]^{\frac{1}{3}} \\
\operatorname{Per}=\frac{4 S+B}{B h}
\end{gathered}
$$

If $u_{L, E f f} \leq 0.255, \mathrm{~K}_{3}=0.38548, \mathrm{~A}_{5}=0.17954, \mathrm{~A}_{6}=0.36739, \mathrm{~K}_{4}=0.02048$

If $0.255 \leq u_{L, E f f} \leq 0.36, \mathrm{~K}_{3}=1.0467, \mathrm{~A}_{5}=0.056825, \mathrm{~A}_{6}=0.5867, \mathrm{~K}_{4}=0.03956$

$$
\text { If } u_{L, E f f} \geq 0.255, \mathrm{~K}_{3}=1.8229, \mathrm{~A}_{5}=-0.04219, \mathrm{~A}_{6}=0.7191, \mathrm{~K}_{4}=0.04258
$$

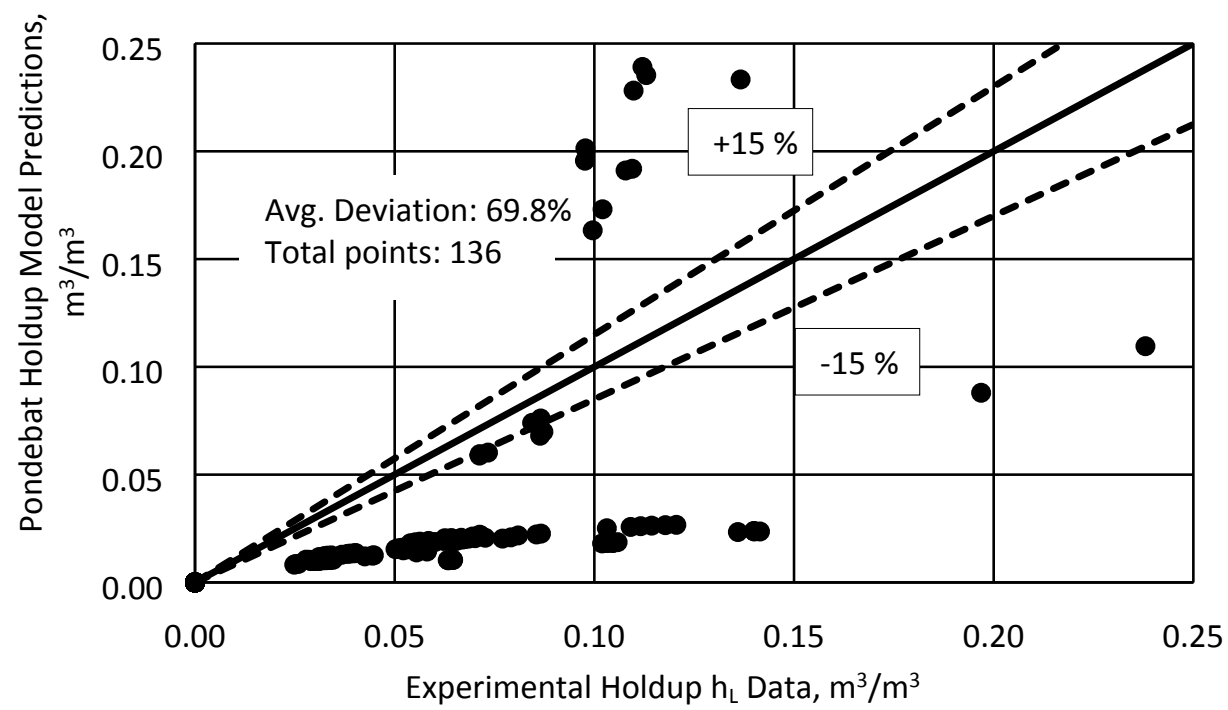

Fig. S10. Pondebat preloading region model predictions vs experimental liquid holdup data 


\section{Spiegel Model ${ }^{11}$ :}

$$
\begin{gathered}
h_{L}=\left(c a_{d}^{0.83} u_{L}^{x}\left(\frac{\mu_{L}}{\mu_{\text {Water }}}\right)^{0.25}\right) \\
c=0.0169 \text { and } x=0.37 \text { if } u_{L}<40 \\
c=0.0075 \text { and } x=0.59 \text { if } u_{L}>40
\end{gathered}
$$

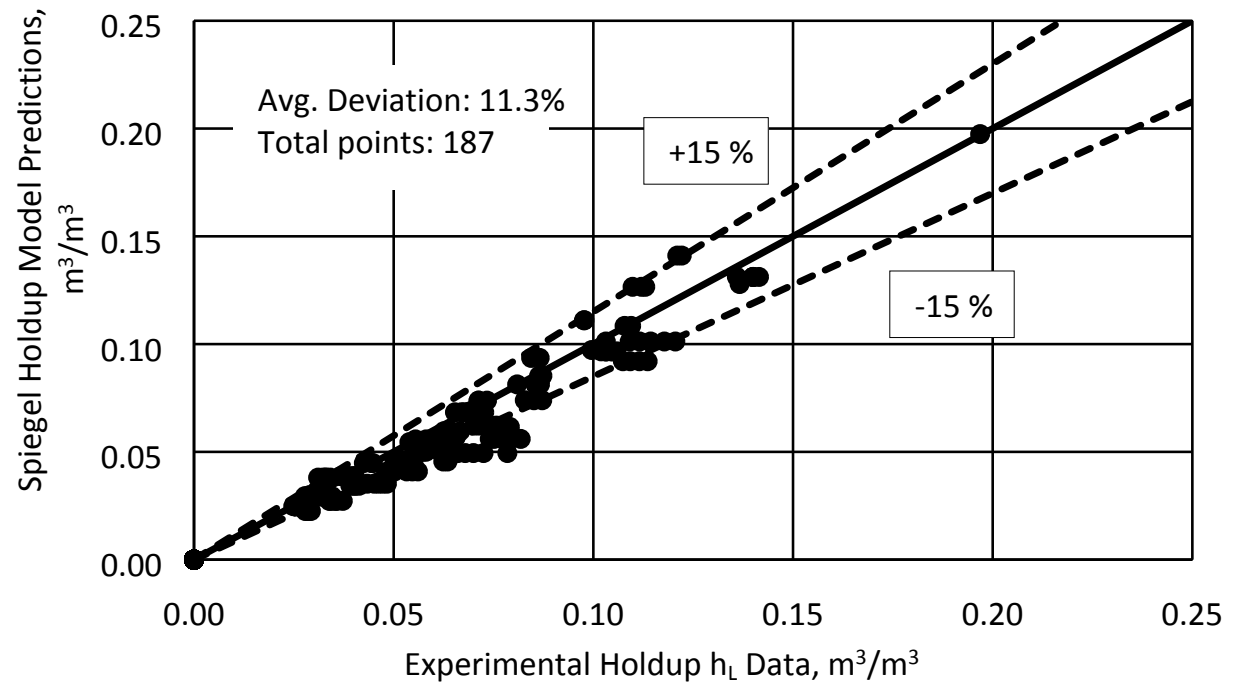

Fig. S11. Spiegel preloading region model predictions vs experimental liquid holdup data 


\section{SRP Model ${ }^{12}$ :}

$$
\begin{aligned}
\left(\frac{\Delta P}{\Delta Z}\right)_{d r y} & =\left(\frac{\rho_{G}}{\rho_{(\text {air }, 1 \text { bar })}}\right)^{0.4}\left(\frac{0.177 \rho_{G} U_{G S}^{2}}{S \epsilon^{2}(\sin \theta)^{2}}+\frac{88.77 u_{G} U_{G S}}{S^{2} \epsilon \sin \theta}\right) \\
F_{t} & =\frac{a_{e}}{a_{p}}=\frac{\left(W e_{L} F r_{L}\right)^{0.15} 29.12 S^{0.36}}{R e_{L}^{0.2} \epsilon^{0.6}(1-0.93 \cos \gamma)(\sin \theta)^{0.3}}
\end{aligned}
$$

Solve the below equations,

$$
\begin{gathered}
\left.h_{L}=\left[\frac{4 F_{t}}{S}\right]^{\frac{2}{3}} \mid \frac{3 \mu_{L} U_{L s}}{\rho_{L} \in \sin \theta\left[\left(\frac{\rho_{L}-\rho_{G}}{\rho_{L}}\right)\left(1-\frac{\left(\frac{\Delta P}{\Delta Z}\right)_{\text {new }}}{\left.\left(\frac{\Delta P}{\Delta Z}\right)_{\text {flood }}\right)}\right)\right.}\right)^{\frac{\Delta P}{\Delta Z}}=\frac{\left(\frac{\Delta P}{\Delta Z}\right)_{d r y}^{\frac{1}{3}}}{\left[1-(0.614+71.35 S) h_{L}\right]^{5}} \\
\left(\frac{\Delta P}{\Delta Z}\right)_{\text {flood }}=1025 \mathrm{~Pa} / \mathrm{m}
\end{gathered}
$$

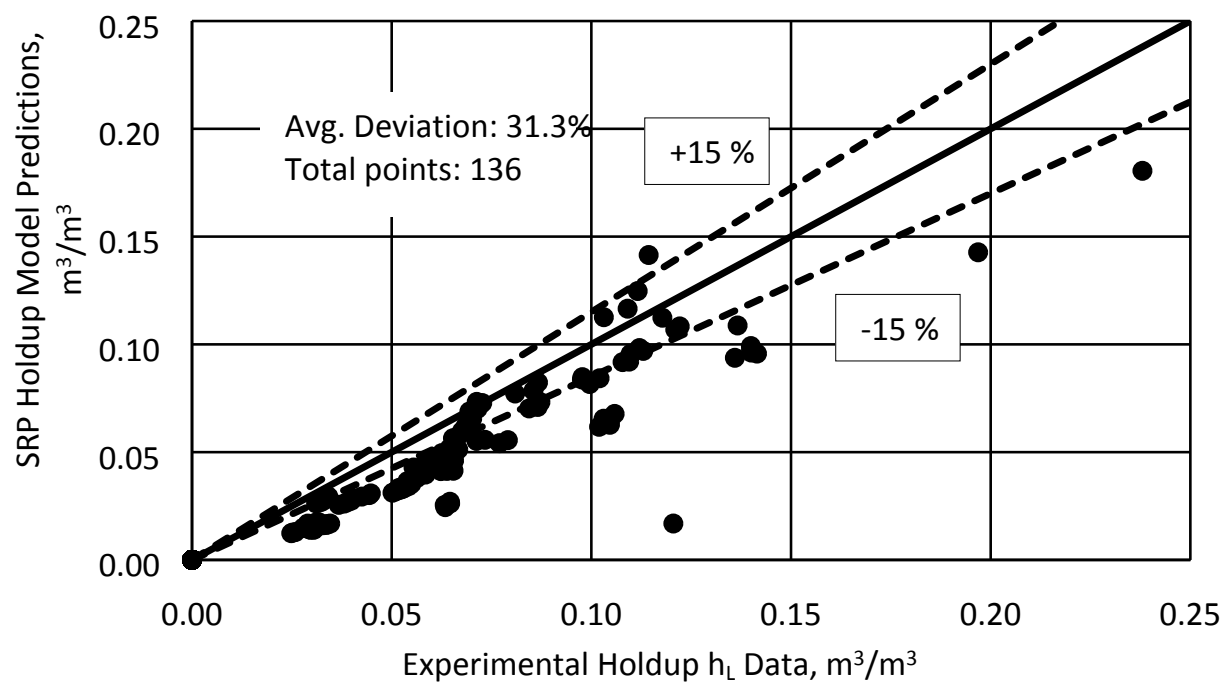


Fig. S12. SRP preloading region model predictions vs experimental liquid holdup data

\section{Stichlmair Model ${ }^{13}$ :}

$$
h_{L}=0.555\left(U_{L}^{2} \frac{a}{g \varepsilon^{4.65}}\right)^{\frac{1}{3}}
$$

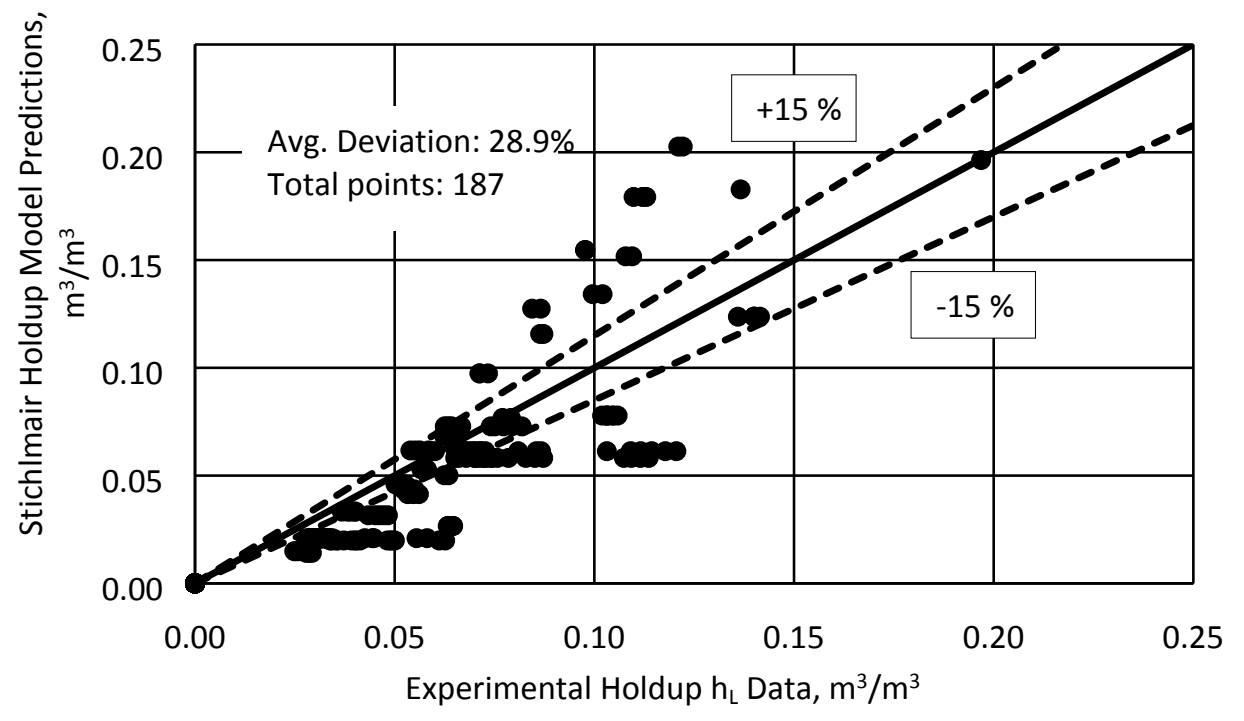

Fig. S13. Stichlmair preloading region model predictions vs experimental liquid holdup data 


\section{Valenz Model ${ }^{14}$ :}

$$
\begin{gathered}
h_{L}=0.6844 F i^{0.5296}\left(\frac{3}{\sin ^{2} \alpha}\right)^{0.877}\left(d_{e q} a_{p}\right)^{2.405} \\
F i=\frac{\eta_{L} u_{L}}{d_{e q}^{2} \rho_{L} g} \\
d_{e q}=B h\left[\frac{1}{B+2 S}+\frac{1}{2 S}\right]
\end{gathered}
$$

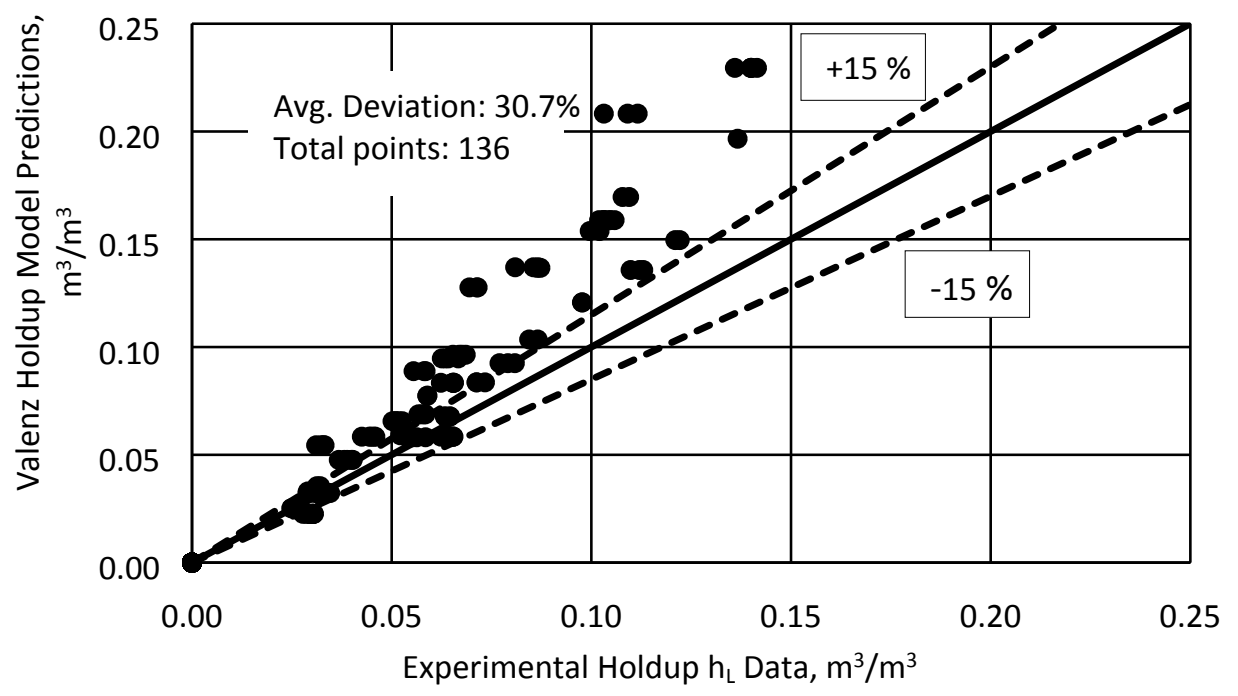

Fig. S14. Valenz preloading region model predictions vs experimental liquid holdup data 


\section{Appendix 2: Preloading Liquid Holdup Data without packing surface area information}

Table S1. Summary of packing data without packing surface area information

\begin{tabular}{|c|c|c|c|c|c|c|c|}
\hline Database & Packing & $\begin{array}{c}\text { Specific Effective } \\
\text { area, } \\
\text { (Approximated) } \\
\text { m2/m3 }\end{array}$ & $\begin{array}{c}\text { Void } \\
\text { fraction, } \\
\varepsilon \\
\end{array}$ & $\mathbf{s}, \mathbf{m m}$ & Preloading & Loading & System \\
\hline \multirow{7}{*}{ Koch ${ }^{12}$} & Gempak 1A & 115 & 96 & 36 & 8 & 7 & \multirow{7}{*}{ Air/Water } \\
\hline & Gempak2A & 220 & 95 & 18 & 6 & 7 & \\
\hline & Gempak 4A & 453 & 91 & 9 & 5 & 11 & \\
\hline & Flexipac 1 & 453 & 91 & 9 & 5 & 10 & \\
\hline & Flexipac 2 & 220 & 95 & 18 & 9 & 15 & \\
\hline & Flexipac 3 & 115 & 96 & 36 & 15 & 13 & \\
\hline & Flexipac 4 & 55 & 98 & 72 & 16 & 12 & \\
\hline \multicolumn{2}{|c|}{ Total Points } & & & & 64 & 75 & \\
\hline
\end{tabular}

Table S2. Summary of literature holdup model performance using experimental liquid holdup data (with approximated packing surface area) in preloading region

\begin{tabular}{|c|c|c|c|c|c|c|c|c|c|c|}
\hline \multirow[b]{2}{*}{ Database } & \multirow[b]{2}{*}{ Packing } & \multicolumn{9}{|c|}{ Mean Absolute Relative Error, \% } \\
\hline & & $\begin{array}{c}\text { OkState Model } \\
\text { (Eqn. 8) }\end{array}$ & $\begin{array}{c}\text { Alix } \\
1\end{array}$ & $\begin{array}{c}\text { Billet } \\
15\end{array}$ & $\begin{array}{c}\text { Delft } \\
4\end{array}$ & $\begin{array}{c}\text { Mackowiak } \\
8 \\
\end{array}$ & $\begin{array}{c}\text { Spiegel } \\
11\end{array}$ & $\begin{array}{c}\text { SRP } \\
12\end{array}$ & $\begin{array}{c}\text { Stichlmair } \\
13\end{array}$ & $\begin{array}{c}\text { Valenz } \\
14 \\
\end{array}$ \\
\hline \multirow{7}{*}{ Koch $^{12}$} & Gempak 1A & 23.6 & 359.3 & NA & NA & 86.5 & 35.9 & 37.7 & 155.3 & 70.3 \\
\hline & Gempak2A & 9.0 & 82.3 & NA & NA & 42.5 & 24.6 & 9.5 & 73.7 & 75.1 \\
\hline & Gempak 4A & 19.5 & 10.8 & NA & NA & 62.0 & 81.4 & 39.4 & 101.0 & 136.8 \\
\hline & Flexipac 1 & 4.0 & 20.4 & NA & NA & 23.2 & 34.0 & 5.2 & 26.5 & 124.5 \\
\hline & Flexipac 2 & 9.1 & 64.7 & NA & NA & 31.3 & 16.4 & 4.9 & 66.0 & 62.9 \\
\hline & Flexipac 3 & 14.5 & 308.5 & NA & NA & 68.9 & 24.5 & 28.8 & 138.0 & 56.6 \\
\hline & Flexipac 4 & 14.7 & 545.5 & NA & NA & 33.7 & 21.2 & 2.3 & 112.3 & 21.0 \\
\hline \multicolumn{2}{|c|}{ Average Deviation } & 14.1 & 272.8 & NA & NA & 50.4 & 29.1 & 17.1 & 106.0 & 63.6 \\
\hline
\end{tabular}

- Lowest error for each packing is highlighted

- Billet model error was reported as NA due to lack of packing specific constants

- For models requiring specific packing dimensions, errors are displayed as NA's where the packing information was not available

$$
\text { MARE, } \%=\frac{(\text { Measured Holdup }- \text { Predicted Holdup })}{(\text { Measured Holdup })} \times 100
$$




\section{Alix-1 Model ${ }^{1}$ :}

$$
\begin{gathered}
h_{L}=h_{L_{0}}+K^{P} \frac{\Gamma}{\rho_{L}}\left(\frac{\mu_{L}}{\mu_{w}}\right)^{\frac{1}{3}} \\
\Gamma=\rho_{L} \frac{V_{S L}}{a_{g}} \\
K^{P}=691\left[\mathrm{~s} \mathrm{~m}^{-2}\right] \\
h_{L_{0}}=6.3 \%
\end{gathered}
$$

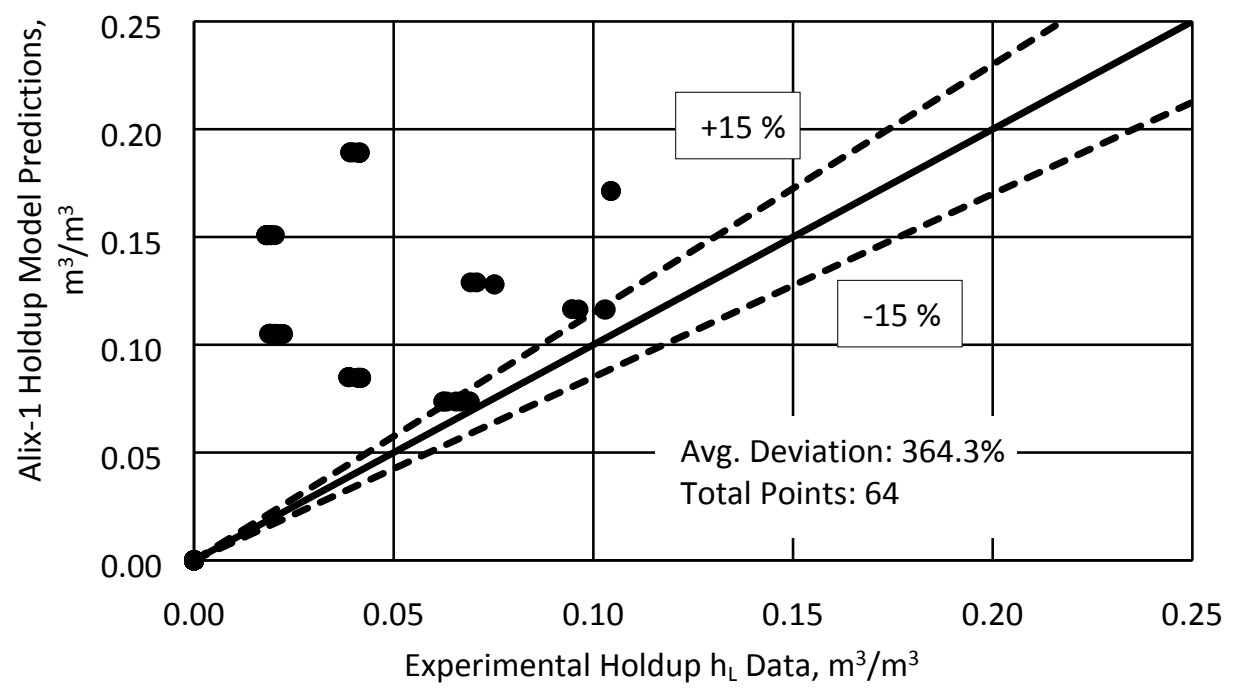

Fig. S15. Alix-1 preloading region model predictions vs experimental liquid holdup data 


\section{Alix-2 Model 1:}

$$
\begin{gathered}
h_{L}=h_{L_{0}}+C_{1} \Gamma^{0.4}\left(\frac{\mu_{L}}{\mu_{w}}\right)^{\frac{1}{3}} \\
R e_{L}=\frac{4 \Gamma}{\mu_{L}}=\frac{4 \rho_{L} V_{S L}}{a_{g} \mu_{L}} \\
C_{1}=0.2683\left[\mathrm{~s} \mathrm{~m}^{-2}\right] \\
R e_{L_{0}}=\frac{4 \Gamma_{0}}{\mu_{L}}=800 \\
h_{L_{0}}=0.032 \frac{\Gamma}{\Gamma_{0}} \text { for } \operatorname{Re}_{L} \leq \operatorname{Re}_{L_{0}} \\
h_{L_{0}}=0.032 \text { for } \operatorname{Re}_{L}>\operatorname{Re}_{L_{0}}
\end{gathered}
$$

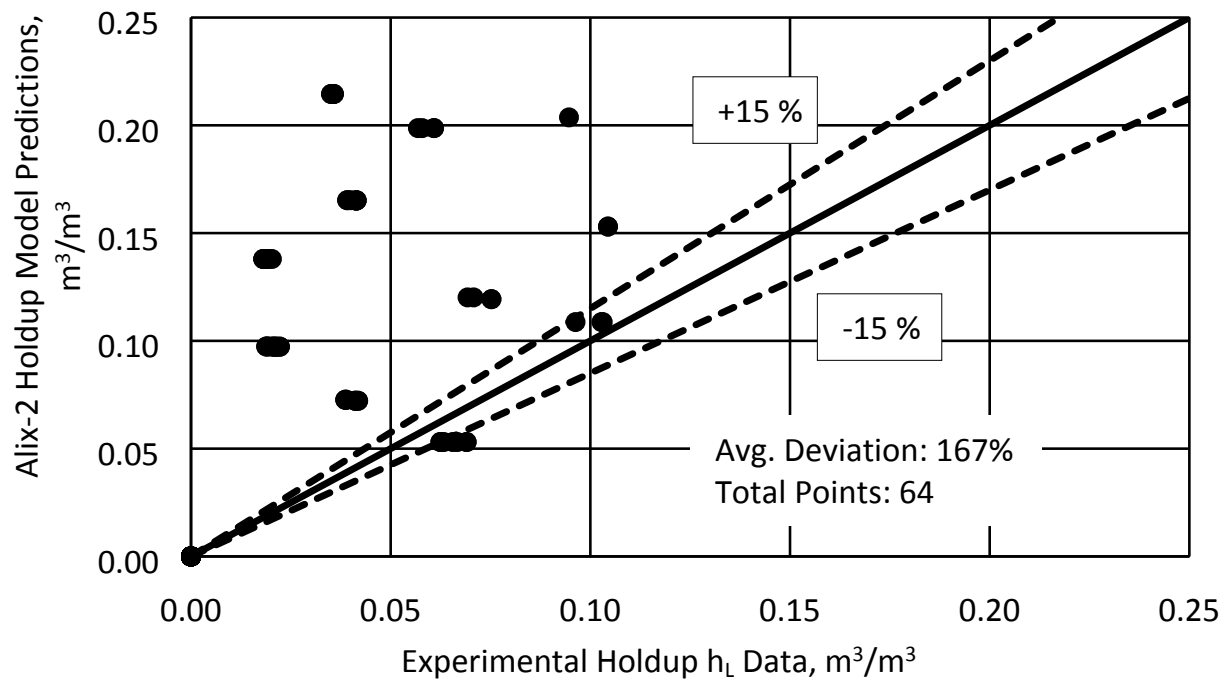

Fig. S16. Alix-2 preloading region model predictions vs experimental liquid holdup data 


\section{Gualito Model ${ }^{6}$ :}

$$
\begin{gathered}
\left(\frac{\Delta P}{\Delta Z}\right)_{d r y}=\left(\frac{\rho_{G}}{\rho_{(\text {air }, 1 \text { bar })}}\right)^{0.4}\left(\frac{0.177 \rho_{G} U_{G S}^{2}}{S \epsilon^{2}(\sin \theta)^{2}}+\frac{88.77 u_{G} U_{G S}}{S^{2} \epsilon \sin \theta}\right) \\
F_{t}=\frac{a_{e}}{a_{p}}=\frac{\left(W e_{L} F r_{L}\right)^{0.15} 29.12 S^{0.36}}{R e_{L}^{0.2} \epsilon^{0.6}(1-0.93 \cos \gamma)(\sin \theta)^{0.3}} \\
\left(\frac{\Delta P}{\Delta Z}\right)_{\text {flood }}=1500+65000 U_{L S}
\end{gathered}
$$

Solve below equations,

$$
\begin{aligned}
& h_{L}=\left[\frac{4 F_{t}}{S}\right]^{\frac{2}{3}}\left(\frac{3 \mu_{L} U_{L s}}{\rho_{L} \epsilon \sin \theta\left[\left(\frac{\rho_{L}-\rho_{G}}{\rho_{L}}\right)\left(1-\frac{\left(\frac{\Delta P}{\Delta Z}\right)_{\text {new }}}{\left.\left(\frac{\Delta P}{\Delta Z}\right)_{\text {flood }}\right)}\right)\right.}\right)^{\frac{1}{3}} \\
& \frac{\Delta P}{\Delta Z}=\frac{\left(\frac{\Delta P}{\Delta Z}\right)_{d r y}}{\left[1-(0.614+71.35 S) h_{L}\right]^{5}}
\end{aligned}
$$




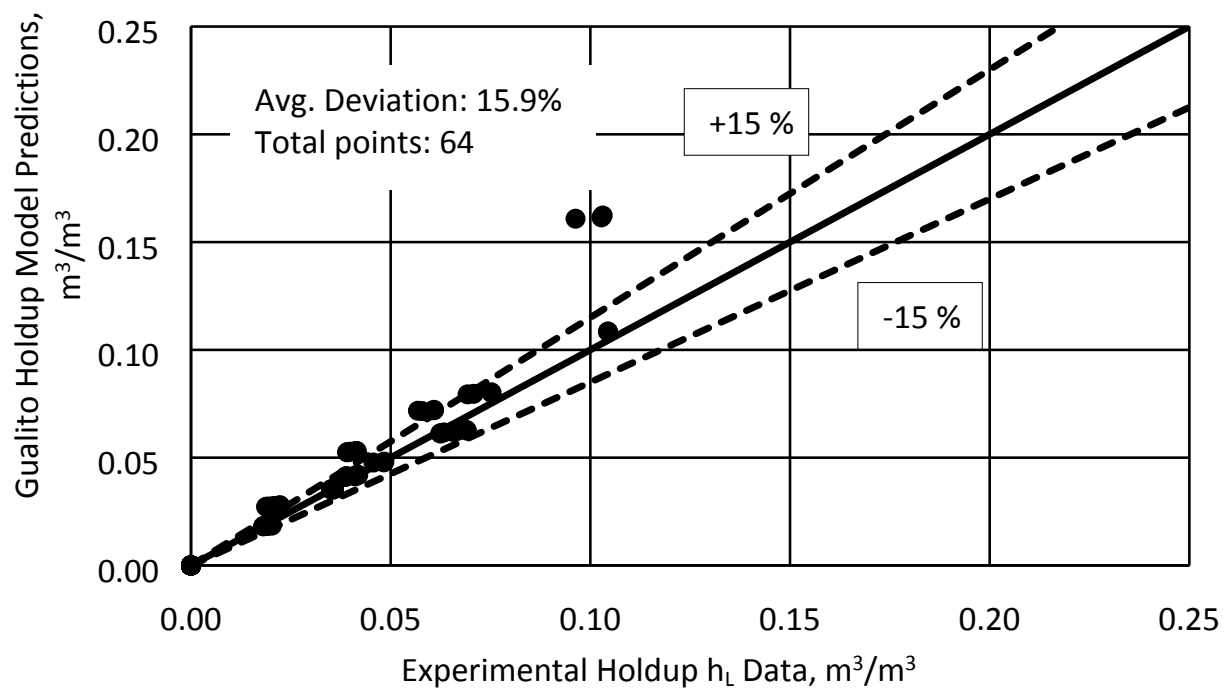

Fig. S17. Gualito preloading region model predictions vs experimental liquid holdup data

\section{Mackowiak-2 Model ${ }^{8}$ :}

$$
h_{L}=0.465\left(\frac{u_{L}^{2} a}{g}\right)^{\frac{1}{3}}
$$

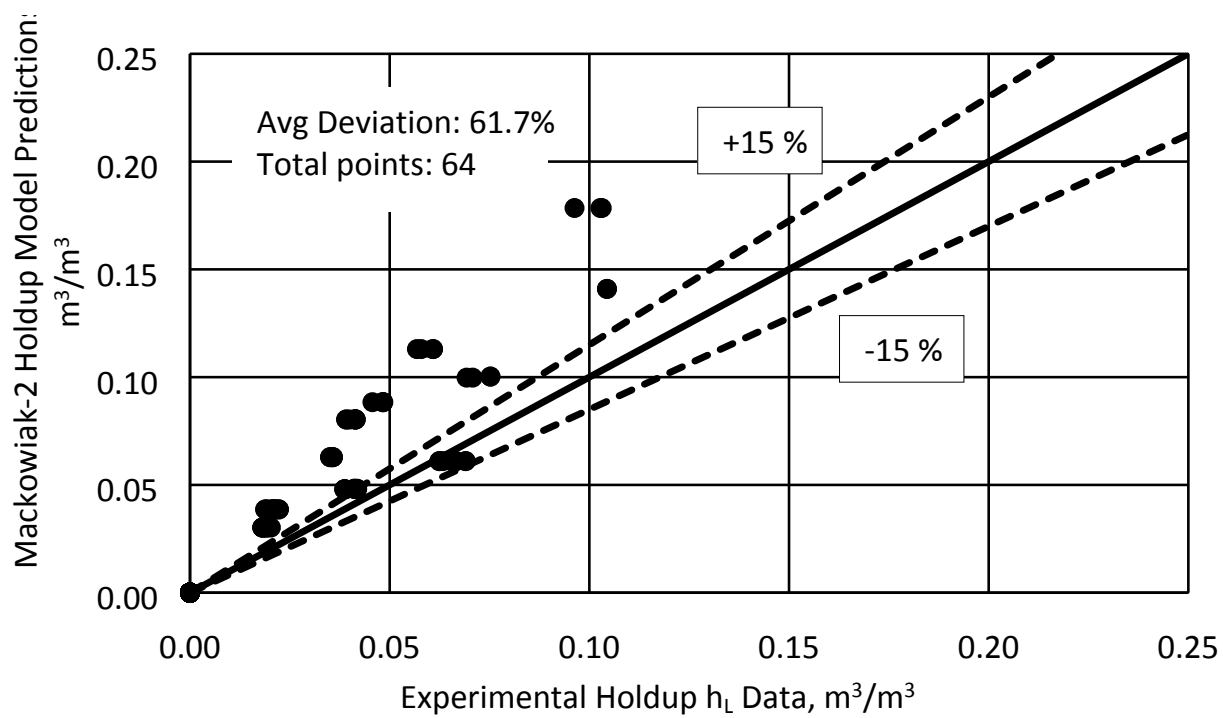


Fig. S18. Mackowiak-2 preloading region model predictions vs experimental liquid holdup data

\section{Mackowiak Model ${ }^{9}$ :}

$$
\begin{gathered}
d_{P}=\frac{6(1-\varepsilon)}{a} \\
B_{L}=\left(\frac{\mu_{L}}{g^{2} \rho_{L}}\right)^{\frac{1}{3}}\left(\frac{u_{L}}{\varepsilon}\right)\left(\frac{1-\varepsilon}{\varepsilon d_{P}}\right) \\
h_{L}=2.2 B_{L}^{0.5}
\end{gathered}
$$




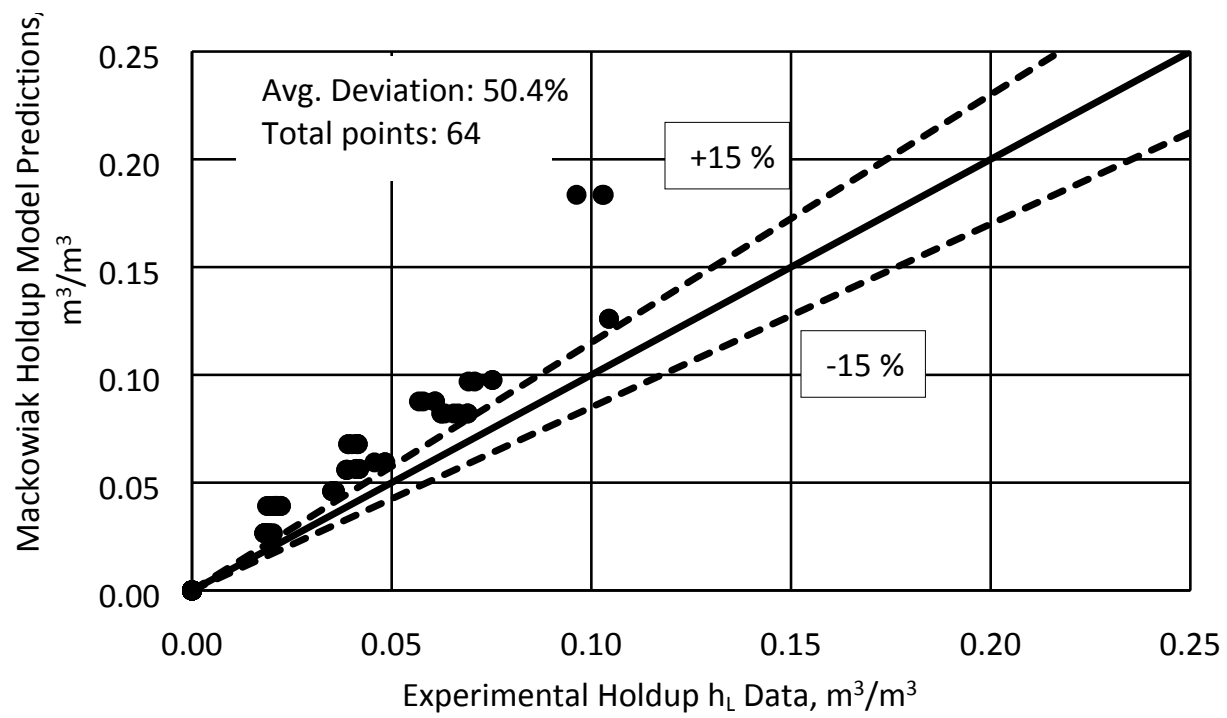

Fig. S19. Mackowiak-2 preloading region model predictions vs experimental liquid holdup data

\section{Newly Developed OkState Preload Model:}




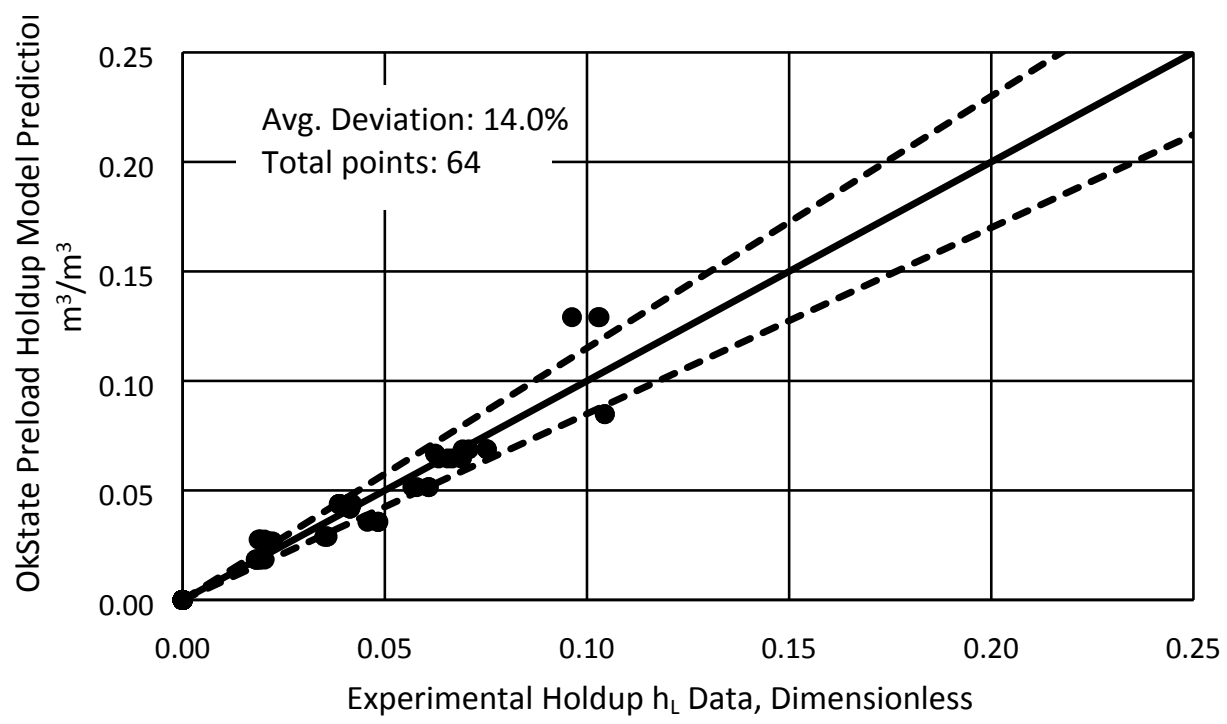

Fig. S20. OkState preload region model predictions vs experimental liquid holdup data

\section{Spiegel Model ${ }^{11}$ :}




$$
\begin{gathered}
h_{L}=\left(c a_{d}^{0.83} u_{L}^{x}\left(\frac{\mu_{L}}{\mu_{\text {Water }}}\right)^{0.25}\right) \\
c=0.0169 \text { and } x=0.37 \text { if } u_{L}<40 \\
c=0.0075 \text { and } x=0.59 \text { if } u_{L}>40
\end{gathered}
$$

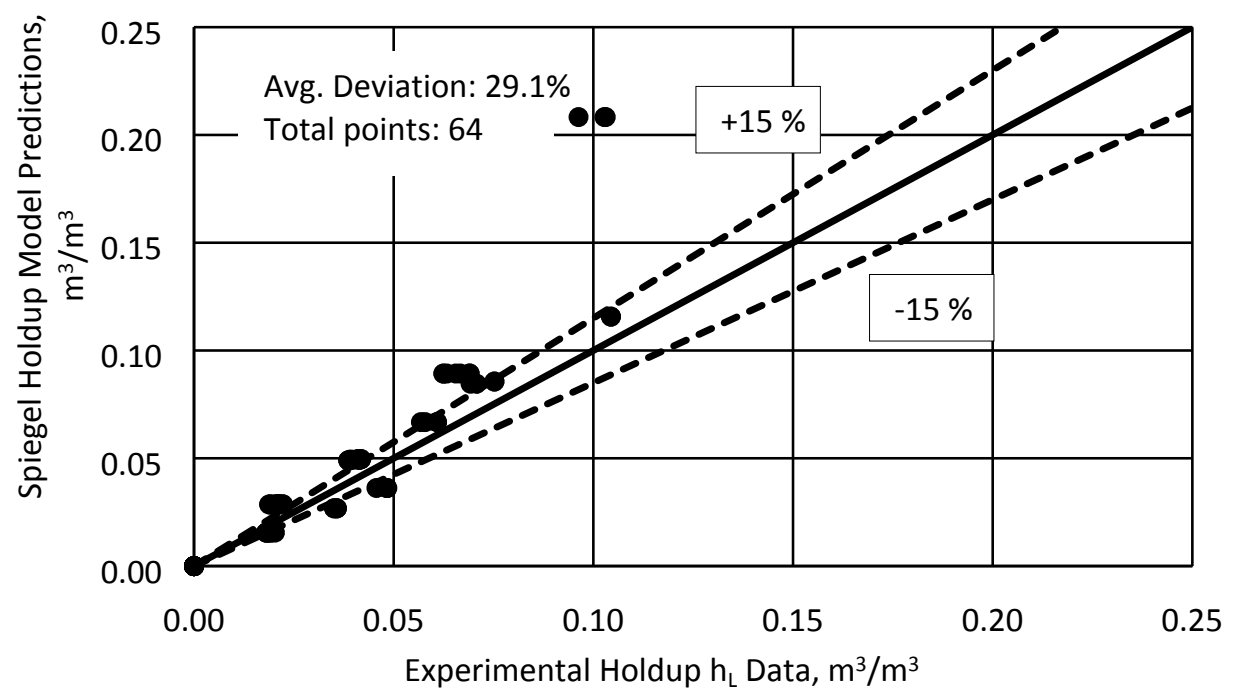

Fig. S21. Spiegel preloading region model predictions vs experimental liquid holdup data

\section{SRP Model 12:}




$$
\begin{aligned}
\left(\frac{\Delta P}{\Delta Z}\right)_{d r y} & =\left(\frac{\rho_{G}}{\rho_{(\text {air }, 1 \text { bar })}}\right)^{0.4}\left(\frac{0.177 \rho_{G} U_{G S}^{2}}{S \epsilon^{2}(\sin \theta)^{2}}+\frac{88.77 u_{G} U_{G S}}{S^{2} \epsilon \sin \theta}\right) \\
F_{t} & =\frac{a_{e}}{a_{p}}=\frac{\left(W e_{L} F r_{L}\right)^{0.15} 29.12 S^{0.36}}{R e_{L}^{0.2} \epsilon^{0.6}(1-0.93 \cos \gamma)(\sin \theta)^{0.3}}
\end{aligned}
$$

Solve the below equations,

$$
\begin{gathered}
\left.h_{L}=\left[\frac{4 F_{t}}{S}\right]^{\frac{2}{3}}\left(\frac{3 \mu_{L} U_{L s}}{\left.\rho_{L} \in \sin \theta \mid\left(\frac{\rho_{L}-\rho_{G}}{\rho_{L}}\right)\left(1-\frac{\left(\frac{\Delta P}{\Delta Z}\right)_{\text {new }}}{\left(\frac{\Delta P}{\Delta Z}\right)_{\text {flood }}}\right)\right]}\right)^{\frac{1}{3}}\right)^{\frac{\Delta P}{\Delta Z}}=\frac{\left(\frac{\Delta P}{\Delta Z}\right)_{d r y}}{\left[1-(0.614+71.35 S) h_{L}\right]^{5}} \\
\left(\frac{\Delta P}{\Delta Z}\right)_{\text {flood }}=1025 P a / m
\end{gathered}
$$

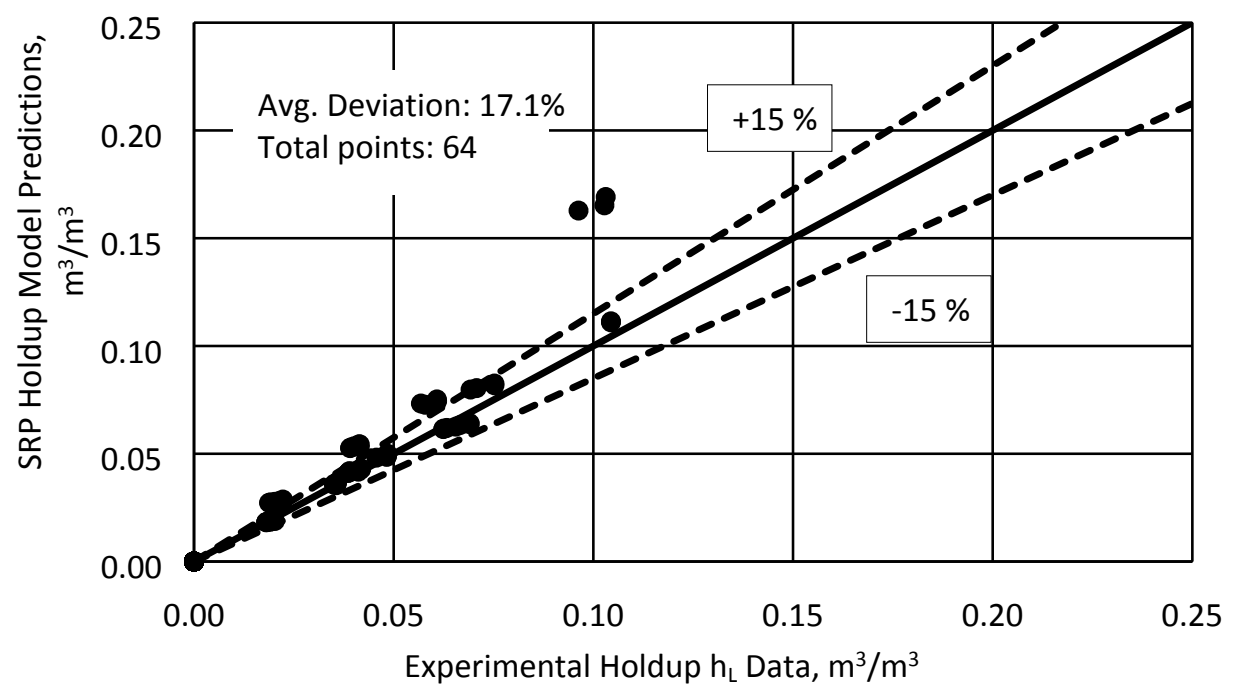

Fig. S22. SRP preloading region model predictions vs experimental liquid holdup data 


\section{Stichlmair Model ${ }^{13}$ :}

$$
h_{L}=0.555\left(U_{L}^{2} \frac{a}{g \varepsilon^{4.65}}\right)^{\frac{1}{3}}
$$

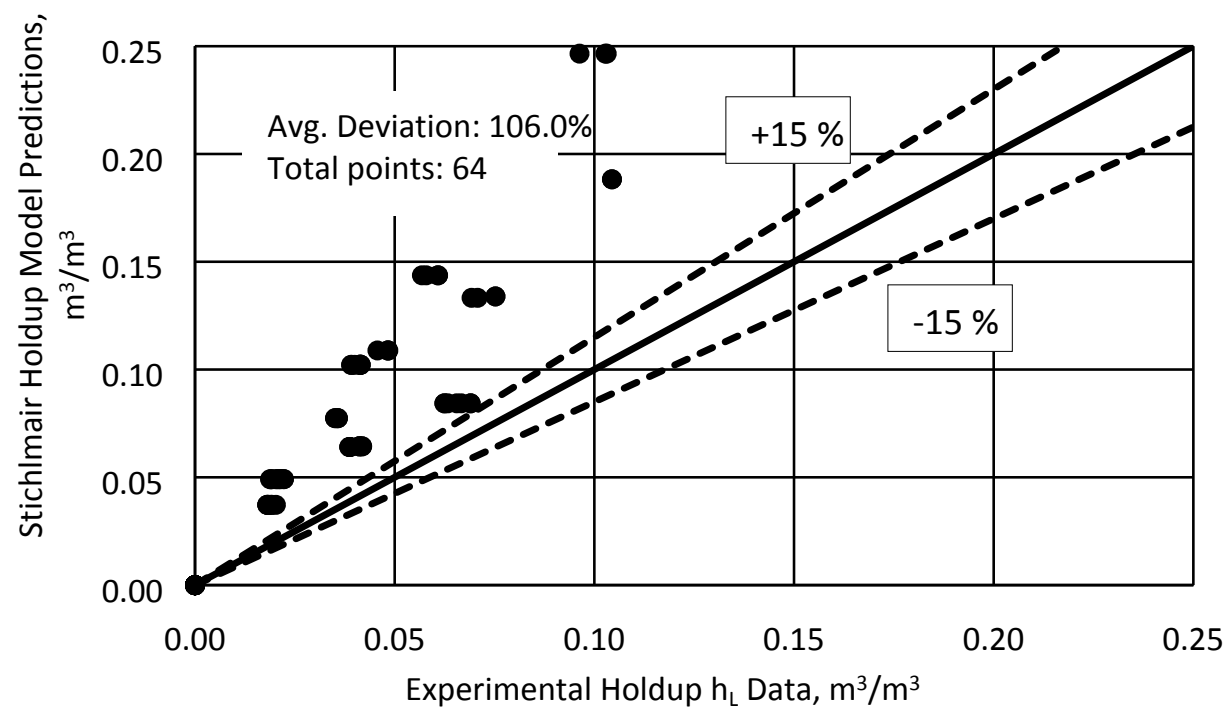

Fig. S23. Stichlmair preloading region model predictions vs experimental liquid holdup data 


\section{Example Problem 2:}

1. A packed column using Mellapak 250X structured packing is being operated under atmospheric conditions with a liquid load of $100 \mathrm{~m} 3 / \mathrm{m} 2 \mathrm{~h}$. Check whether the column is flooded or not when the measured liquid holdup of the column is $0.21 \mathrm{~m} 3 / \mathrm{m} 3$ and the vapor velocity of the column is $2.56 \mathrm{~m} / \mathrm{s}$. Use below properties for the calculations:

$\begin{array}{llll}\text { Water Density } & = & 1000 \mathrm{~kg} / \mathrm{m}^{3} \\ \text { Water Viscosity } & = & 10 \mathrm{cp} \\ \text { Air Density } & = & 1.3 \mathrm{~kg} / \mathrm{m}^{3} \\ \text { Packing Area (a) } & = & 250 \mathrm{~m}^{2} / \mathrm{m}^{3} \\ \text { Void fraction (c) } & = & 98 \mathrm{~m}^{3} / \mathrm{m}^{3} \\ \text { Corrugation angle } & = & 60 \\ \text { Surface tension } & = & 0.072 \mathrm{~N} / \mathrm{m}\end{array}$

Ans: a. Liquid holdup value at flooding:

First calculating the critical liquid holdup using OkState liquid holdup flooding model (equation 5).

$$
h_{L, f l}=2.0 \frac{\mathrm{a}^{0.88} \operatorname{Re}^{0.41}}{(1-\varepsilon)^{0.15}}\left(\frac{\mu_{\mathrm{L}}^{2}}{\rho^{2} \mathrm{gsin} \theta}\right)^{\frac{1}{3}}
$$

Where, 


$$
\begin{gathered}
R e=\frac{u_{L} \rho_{L}}{a \mu_{L}}=\frac{\left(\frac{100}{3600}\right)\left(\frac{m}{s}\right) 1000\left(\frac{\mathrm{kg}}{\mathrm{m}^{3}}\right)}{250\left(\frac{\mathrm{m}^{2}}{\mathrm{~m}^{3}}\right)\left(\frac{10}{10000}\right)(\text { Pa.s })}=111.11 \\
h_{L, f l}=2.0 \frac{250^{0.88} 111.11^{0.41}}{(1-0.98)^{0.15}}\left(\frac{(10 / 10000)^{2}}{1000^{2} 9.81 \sin 60}\right)^{\frac{1}{3}}=0.157 \mathrm{~m}^{3} / \mathrm{m}^{3}
\end{gathered}
$$

Since the experimental liquid holdup $\left(h_{L, \exp }=0.21 \mathrm{~m} 3 / \mathrm{m} 3\right)$ is greater than the model predicted critical value of $0.157 \mathrm{~m}^{3} / \mathrm{m}^{3}$. The column is flooded based on the experimental liquid holdup data.

b. Flood point vapor velocity:

Flood point vapor velocity can be calculated using the OkState flooding velocity model (equation 16).

$$
u_{G, f l}=0.695 \frac{d_{e}^{0.36} \mu^{0.25} \sin \theta}{\sigma^{0.25}}\left(1-h_{L, f l}\right)^{3}\left(\frac{\rho_{L}-\rho_{G}}{\rho_{G}} g\right)^{0.5}
$$

Where,

$$
\begin{gathered}
d_{e}=\frac{4 \varepsilon}{a}=\frac{4 \times 0.98}{250}=0.01568 \mathrm{~m} \\
u_{G, f l}=0.695 \frac{(0.01568)^{0.36}\left(\frac{10}{10000}\right)^{0.25} \sin 60}{0.072^{0.25}}(1-0.157)^{3}\left(\frac{1000-1.3}{1.3} 9.81\right)^{0.5}
\end{gathered}
$$




$$
u_{G, f l}=2.40 \mathrm{~m} / \mathrm{s}
$$

Since the vapor velocity of the column $\left(u_{G}=2.56 \mathrm{~m} / \mathrm{s}\right)$ is greater than the OkState flooding velocity model predictions. The column is considered to be flooded.

\section{Appendix 3}

\section{Liquid Holdup Flooding Model}

\section{Mackowiak Model 16:}

$$
\lambda_{0}=\left(\frac{u_{L}}{u_{V}}\right)_{F L}
$$

When $R e_{L} \geq 2$ :

$$
h_{L, F l}^{O}=\frac{\left(\sqrt{1.44 \lambda_{0}^{2}+0.8 \lambda_{0}\left(1-\lambda_{0}\right)}-1.2 \lambda_{0}\right)}{0.4\left(1-\lambda_{0}\right)}\left[\frac{\mathrm{m}^{3}}{\mathrm{~m}^{3}}\right]
$$

When $e_{L}<2$ :

$$
h_{L, F l}^{O}=\frac{\left(\sqrt{1.254 \lambda_{0}^{2}+0.48 \lambda_{0}\left(1-\lambda_{0}\right)}-1.12 \lambda_{0}\right)}{0.24\left(1-\lambda_{0}\right)}\left[\frac{m^{3}}{m^{3}}\right]
$$




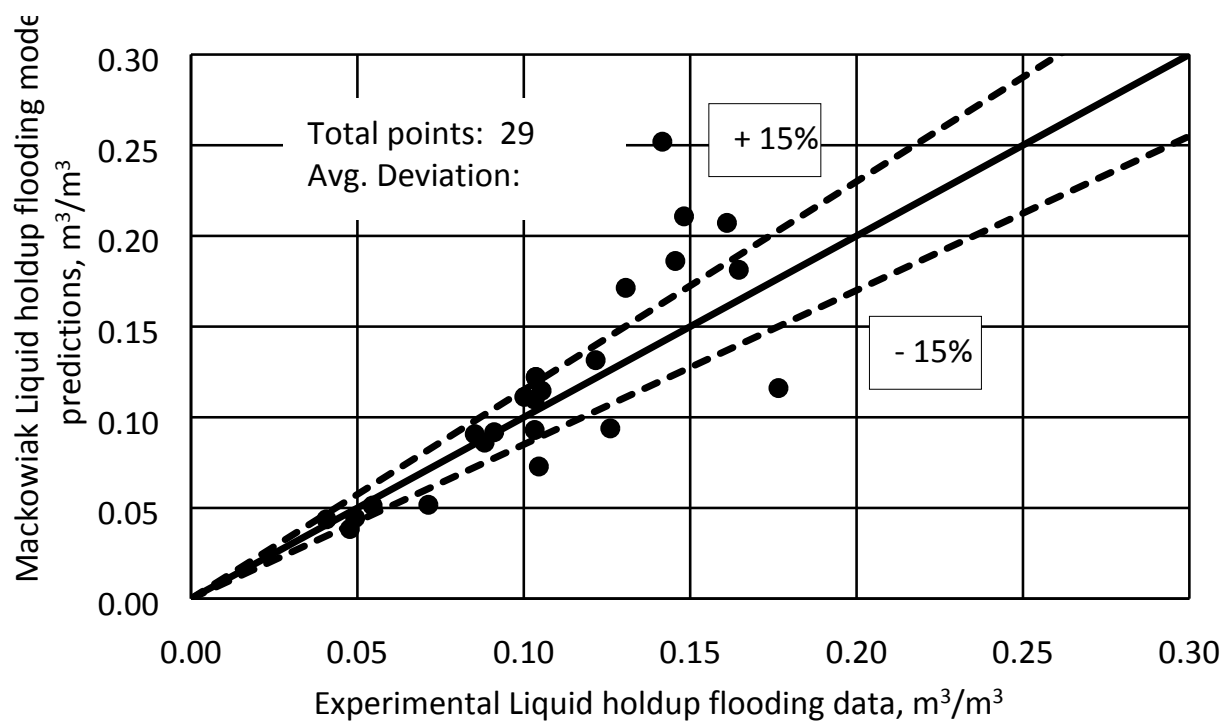

Fig. S24. Mackowiak liquid holdup flooding model predictions vs experimental liquid holdup flooding data

\section{Appendix 4}

\section{Flooding Velocity Models}

\section{Lockett Model ${ }^{17}$ :}

$$
\begin{gathered}
u_{v, f l}=c_{g}\left(\frac{\rho_{l}-\rho_{g}}{\rho_{g}}\right)^{0.5} \\
c_{g}=\left(\frac{N u}{d_{e}}\right)^{2} \\
d_{e}=\left(1+m\left(\frac{\rho_{G}}{\rho_{l}}\right)^{0.5}\left(\frac{\rho_{l}-\rho_{G}}{\rho_{G}}\right)^{0.25}\left(\frac{L}{V}\right)^{0.5}\right. \\
N u=\left(1.57 a^{\left.-0.25\left(\frac{\mu_{l}}{\mu_{w}}\right)^{-0.03}\right)}\right. \\
m=0.78 \exp (0.00058 \mathrm{a})
\end{gathered}
$$




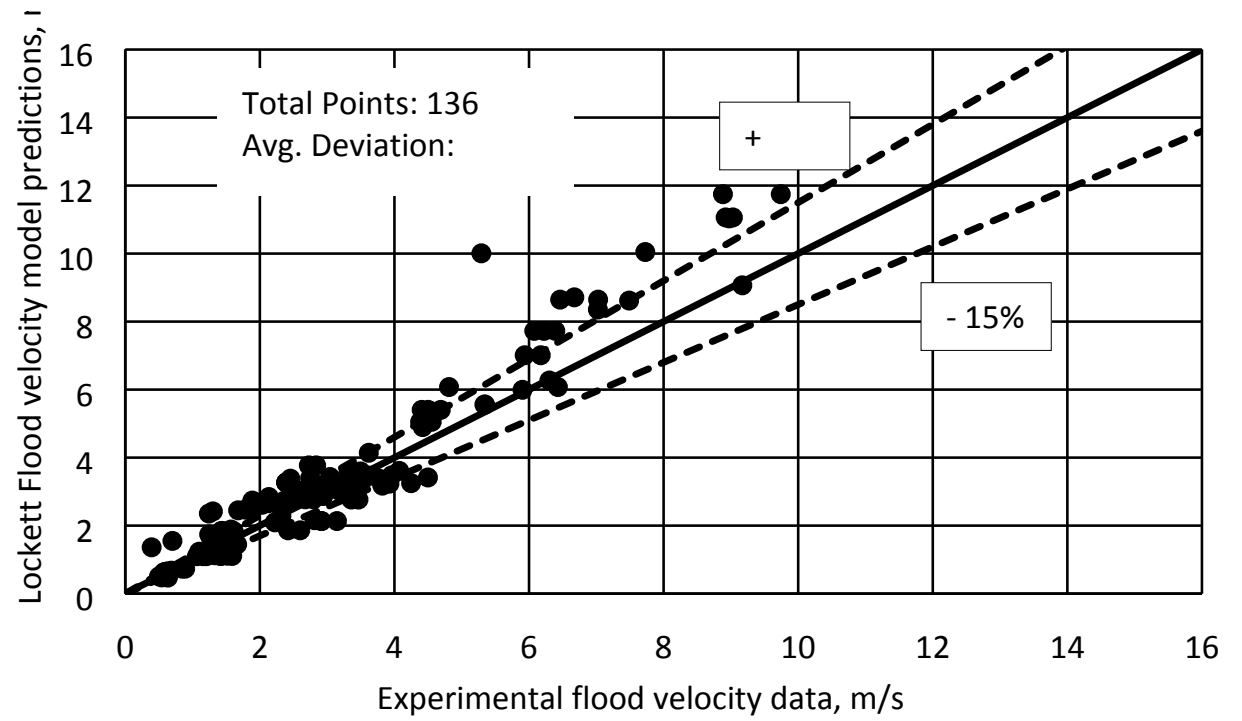

Fig. S25. Lockett flooding velocity model predictions vs experimental flooding velocity data

\section{Kuzniewska Model 18:}

$$
\begin{gathered}
u_{g, f l}=A_{1} \epsilon\left(\frac{a}{\epsilon^{3}}\right)^{B_{1}} \\
A_{1}=A \frac{\left(\frac{\rho_{a} \rho_{l}}{\rho_{g} \rho_{w}}\right)^{0.5}}{\left(\frac{\sigma_{L}}{\sigma_{w}}\right)^{0.25}\left(\frac{\eta_{l}}{\eta_{w}}\right)^{0.02}} \\
B_{1}=B \frac{\left(\frac{v_{l}}{v_{w}}\right)^{0.02}}{\left(\frac{\eta_{g}}{\eta_{a}}\right)^{0.1}\left(\frac{\sigma_{l}}{\sigma_{w}}\right)^{0.15}}
\end{gathered}
$$




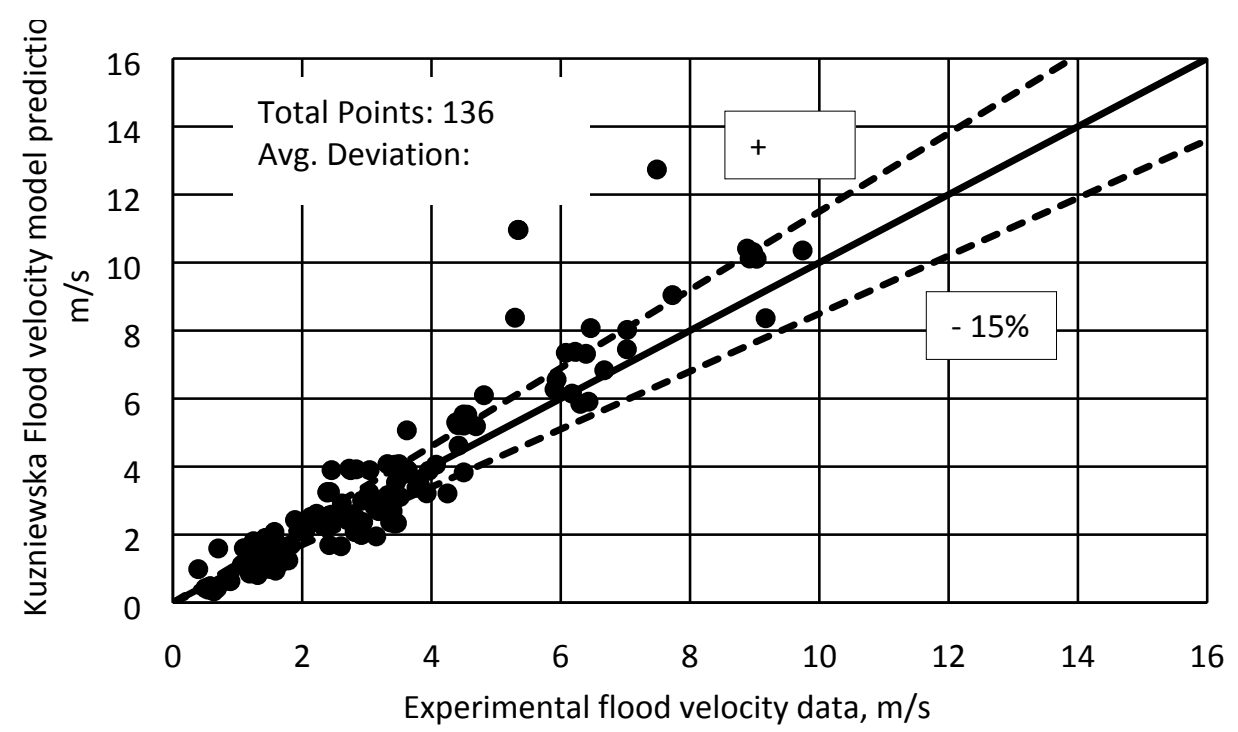

Fig. S26. Kuzniewska flooding velocity model predictions vs experimental flooding velocity data

Appendix 5: Data without packing surface area information

Table S3. Summary of packing data without packing surface area information

\begin{tabular}{|c|c|c|c|c|c|}
\hline Database & Packing & $\begin{array}{c}\text { Effective area, } \\
\text { (Approximated) } \\
\text { m2/m3 }\end{array}$ & $\begin{array}{c}\text { Void } \\
\text { fraction, } \\
\varepsilon \\
\end{array}$ & $\mathbf{s}, \mathbf{m m}$ & System \\
\hline \multirow{6}{*}{ Koch $^{12}$} & Gempak 1A & 115 & 96 & 36 & \multirow{6}{*}{ Air/Water } \\
\hline & Gempak2A & 220 & 95 & 18 & \\
\hline & Gempak 4A & 453 & 91 & 9 & \\
\hline & Flexipac 1 & 453 & 91 & 9 & \\
\hline & Flexipac 2 & 220 & 95 & 18 & \\
\hline & Flexipac 3 & 115 & 96 & 36 & \\
\hline
\end{tabular}




\begin{tabular}{|l|l|l|l|l|l|} 
& Flexipac 4 & 55 & 98 & 72 & \\
\hline
\end{tabular}

Appendix 5.1: Liquid Holdup Flooding Model

1. OkState Liquid holdup flooding model: 


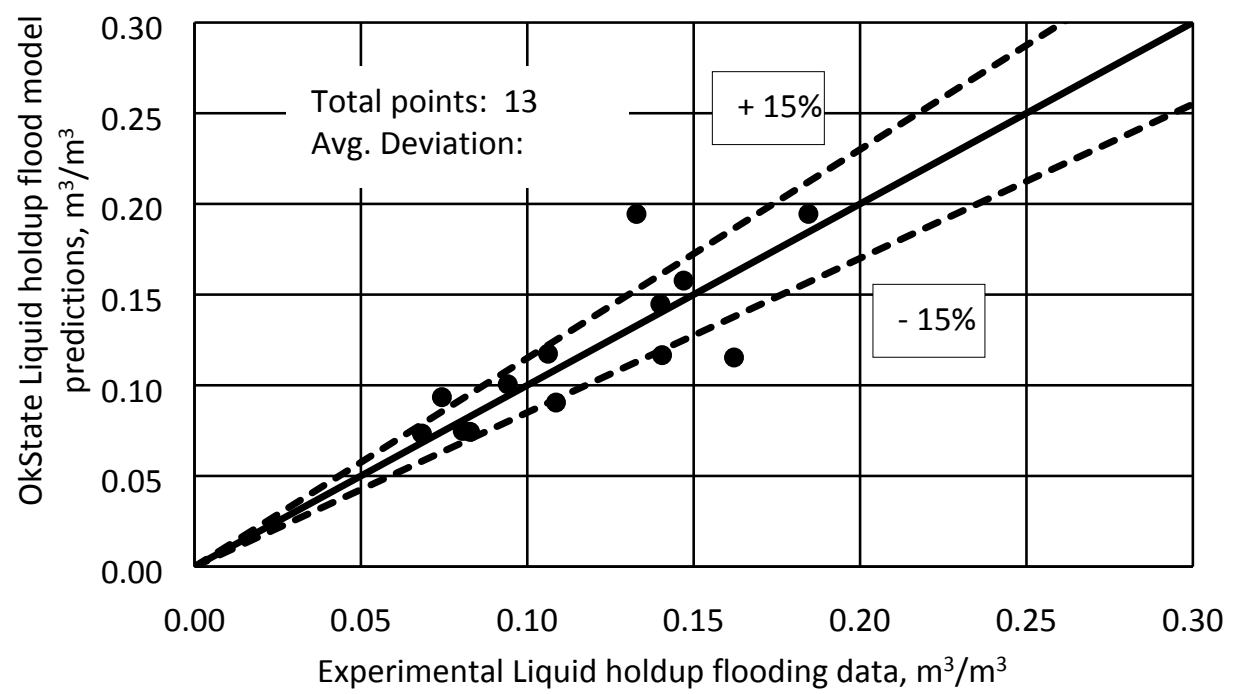

Fig. S27. OkState liquid holdup flooding model predictions vs experimental liquid holdup flooding data

\section{Mackowiak Model ${ }^{16}$ :}

$$
\lambda_{0}=\left(\frac{u_{L}}{u_{V}}\right)_{F L}
$$


When $R e_{L} \geq 2$ :

$$
h_{L, F l}^{O}=\frac{\left(\sqrt{1.44 \lambda_{0}^{2}+0.8 \lambda_{0}\left(1-\lambda_{0}\right)}-1.2 \lambda_{0}\right)}{0.4\left(1-\lambda_{0}\right)}\left[\frac{\mathrm{m}^{3}}{\mathrm{~m}^{3}}\right]
$$

When $e_{L}<2$ :

$$
h_{L, F l}^{O}=\frac{\left(\sqrt{1.254 \lambda_{0}^{2}+0.48 \lambda_{0}\left(1-\lambda_{0}\right)}-1.12 \lambda_{0}\right)}{0.24\left(1-\lambda_{0}\right)}\left[\frac{m^{3}}{m^{3}}\right]
$$

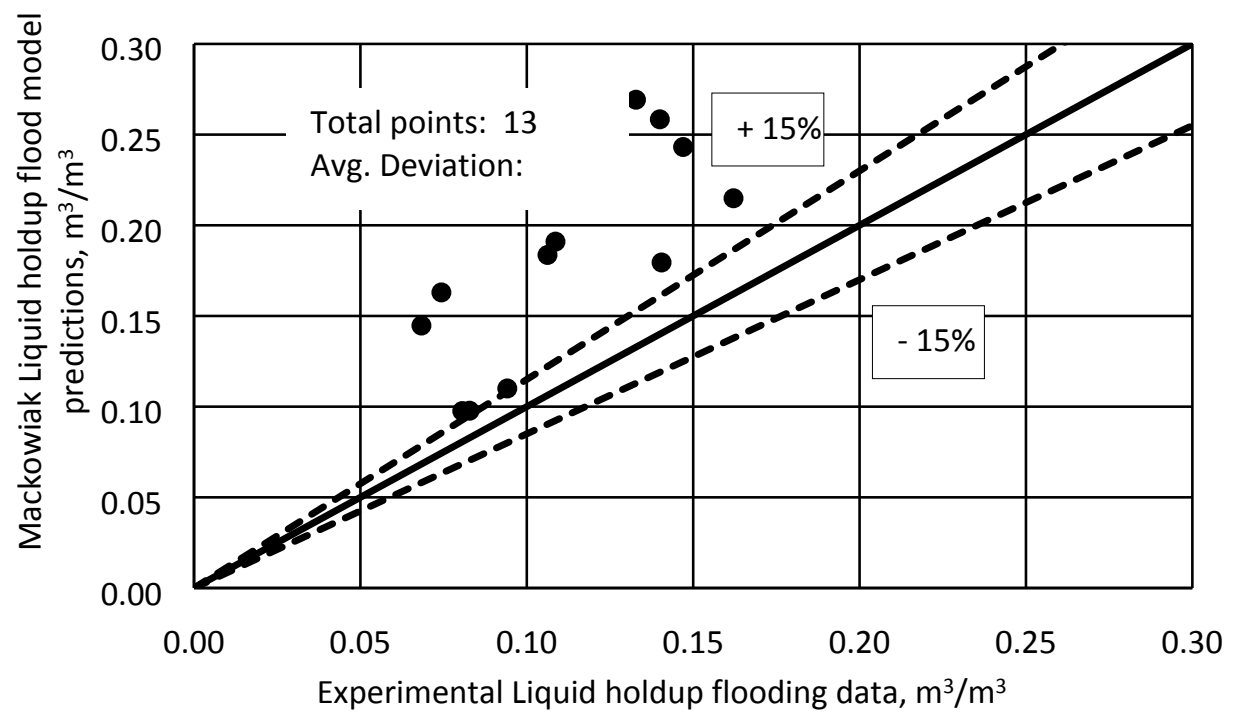

Fig. S28. Mackowiak liquid holdup flooding model predictions vs experimental liquid holdup flooding data

\section{Appendix 5.2: Flooding Velocity Models}

Table S4. Summary of literature flood velocity model performance using experimental flood velocity data (Table 3 ) 


\begin{tabular}{|l|c|}
\hline & $\begin{array}{c}\text { Mean Absolute Relative Error, } \\
\%\end{array}$ \\
\hline OkState & 17.4 \\
\hline Lockett & 32.6 \\
\hline Kuzniewska & 17.4 \\
\hline
\end{tabular}

MARE, $\%=\frac{(\text { Measured Flooding velocity }- \text { Predicted looding velocity })}{(\text { Measured looding velocity })} \times 100$

1. Lockett Model ${ }^{17}$ :

$$
u_{v, f l}=c_{g}\left(\frac{\rho_{l}-\rho_{g}}{\rho_{g}}\right)^{0.5}
$$




$$
\begin{gathered}
c_{g}=\left(\frac{N u}{d_{e}}\right)^{2} \\
d_{e}=\left(1+m\left(\frac{\rho_{G}}{\rho_{l}}\right)^{0.5}\left(\frac{\rho_{l}-\rho_{G}}{\rho_{G}}\right)^{0.25}\left(\frac{L}{V}\right)^{0.5}\right. \\
N u=\left(1.57 a^{-0.25}\left(\frac{\mu_{l}}{\mu_{w}}\right)^{-0.03}\right) \\
m=0.78 \exp (0.00058 \mathrm{a})
\end{gathered}
$$

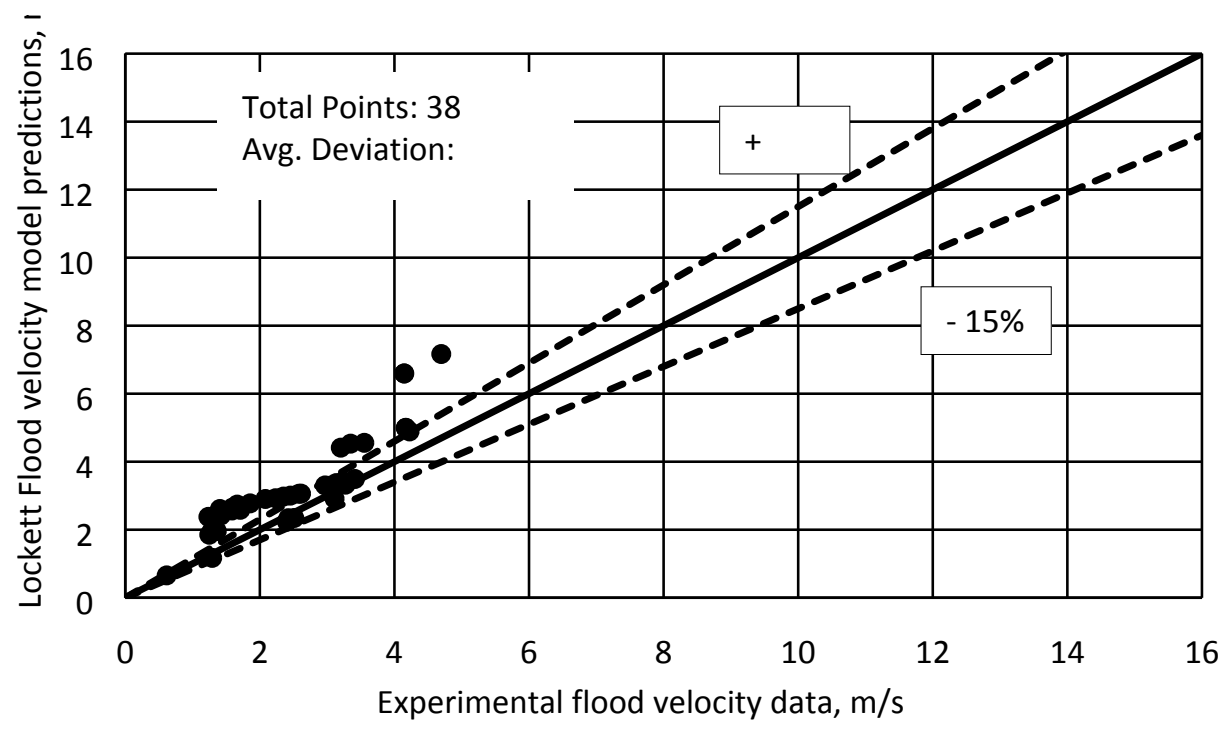

Fig. S29. Lockett flooding velocity model predictions vs experimental flooding velocity data

\section{Kuzniewska Model ${ }^{18}$ :}




$$
\begin{gathered}
u_{g, f l}=A_{1} \epsilon\left(\frac{a}{\epsilon^{3}}\right)^{B_{1}} \\
A_{1}=A \frac{\left(\frac{\rho_{a} \rho_{l}}{\rho_{g} \rho_{w}}\right)^{0.5}}{\left(\frac{\sigma_{L}}{\sigma_{w}}\right)^{0.25}\left(\frac{\eta_{l}}{\eta_{w}}\right)^{0.02}} \\
B_{1}=B \frac{\left(\frac{v_{l}}{v_{w}}\right)^{0.02}}{\left(\frac{\eta_{g}}{\eta_{a}}\right)^{0.1}\left(\frac{\sigma_{l}}{\sigma_{w}}\right)^{0.15}}
\end{gathered}
$$

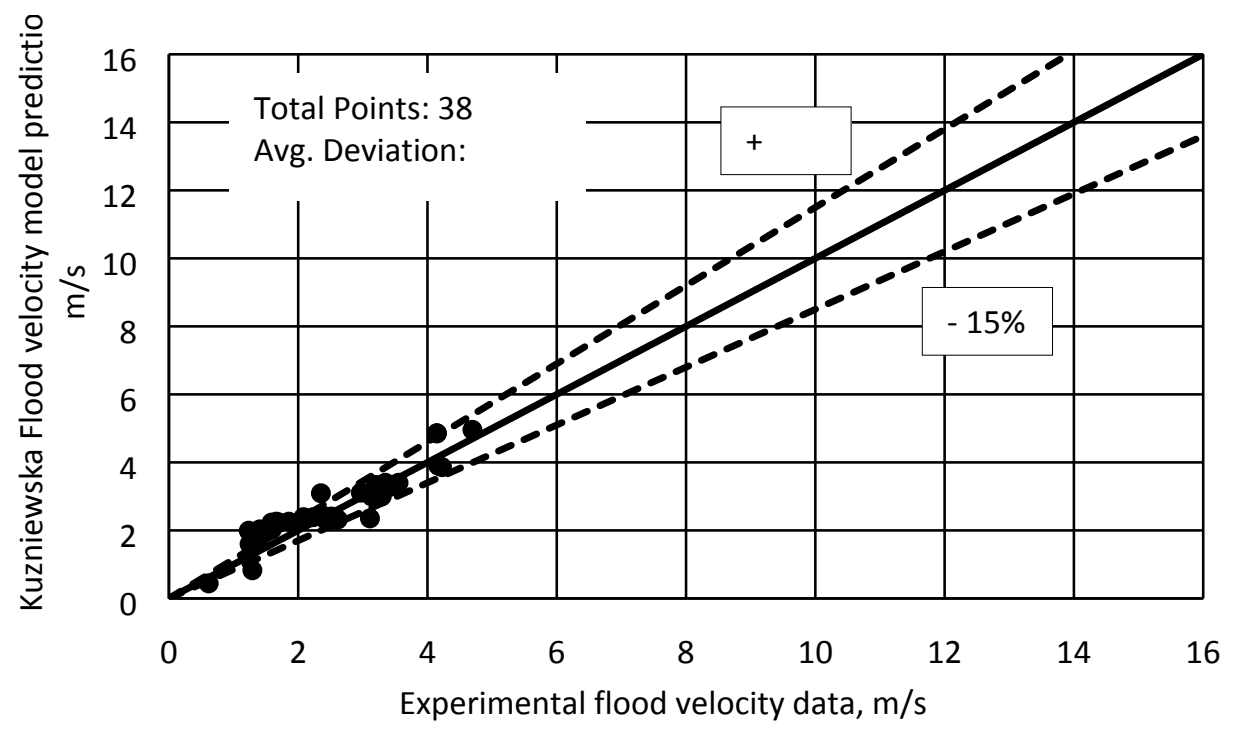

Fig. S30. Kuzniewska flooding velocity model predictions vs experimental flooding velocity data 


\section{OkState Flood velocity model:}

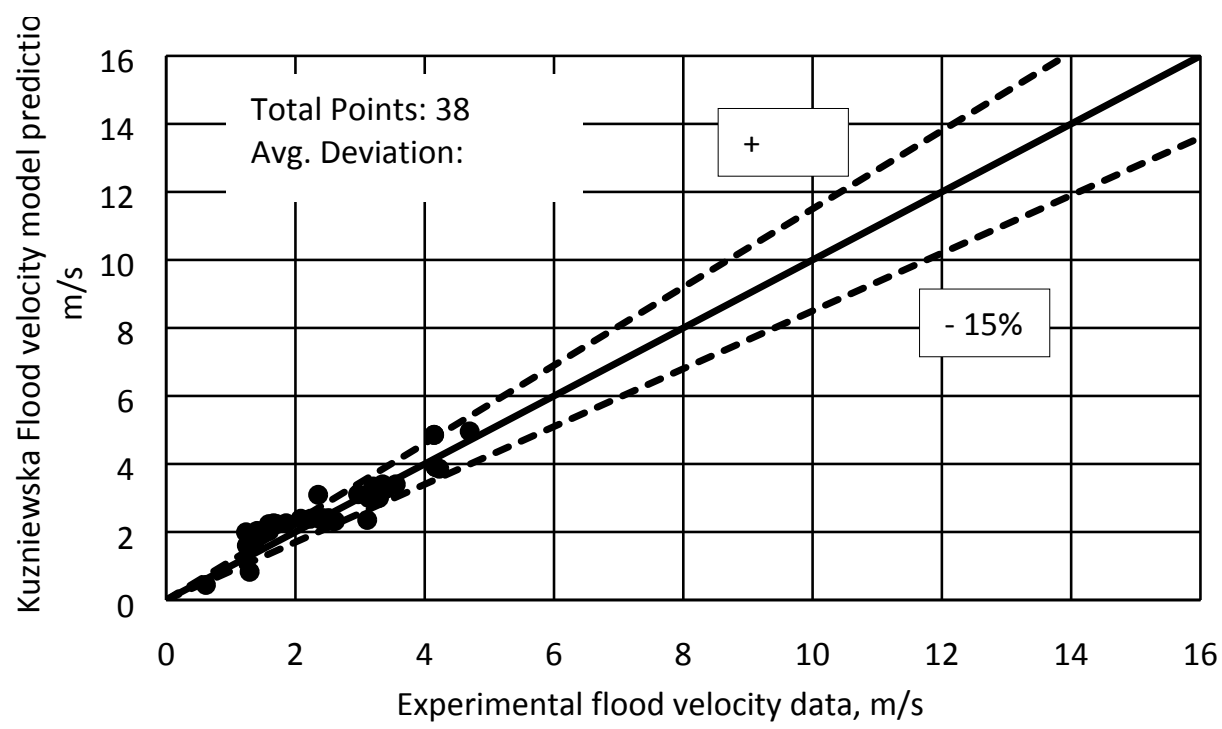

Fig. S31. OkState flooding velocity model predictions vs experimental flooding velocity data 


\section{References:}

1. Alix, P.; Raynal, L., Liquid distribution and liquid hold-up in modern high capacity packings. Chemical Engineering Research and Design 2008, 86 (6), 585-591.

2. Olujic, Z.; Kamerbeek, A. B.; de Graauw, J., A corrugation geometry based model for efficiency of structured distillation packing. Chemical Engineering and Processing 1999, 38 (4-6), 683-695.

3. Olujic, Z., Development of a complete simulation model for predicting the hydraulic and separation performance or distillation columns equipped with structured packings. Chem. Biochem. Eng. Q. 1997, 11 (1), 31-46.

4. Olujic, Z.; Behrens, M.; Colli, L.; Paglianti, A., Predicting the efficiency of corrugated sheet structured packings with large specific surface area. Chem. Biochem. Eng. Q. 2004, 18 (2), 89-96.

5. Olujic, Z.; Jansen, H.; Kaibel, B.; Rietfort, T.; Zich, E., Stretching the Capacity of Structured Packingst. Industrial \& Engineering Chemistry Research 2001, 40 (26), 6172-6180.

6. Gualito, J. J.; Cerino, F. J.; Cardenas, J. C.; Rocha, J. A., Design Method for Distillation Columns Filled with Metallic, Ceramic, or Plastic Structured Packings. Industrial \& Engineering Chemistry Research 1997, 36 (5), 1747-1757.

7. Macías-Salinas, R.; Fair, J. R., Axial mixing in modern packings, gas and liquid phases: I. Singlephase flow. AlChE Journal 1999, 45 (2), 222-239.

8. Mackowiak, J., Pressure drop in irrigated packed columns. Chemical Engineering and Processing: Process Intensification 1991, 29 (2), 93-105.

9. Mackowiak, J., Fluid dynamics of packed columns. Principles of the Fluid Dynamic Design of Columns for Gas/Liquid and Liquid/Liquid Systems, London, New York 2010.

10. Pondebat, C. P., J. L. Huor, M. H. Prevost, M., Hydrodynamics of a gas-liquid column equipped with a structured packing. INSTITUTION OF CHEMICAL ENGINEERS SYMPOSIUM SERIES 1992, VOL 128/2, pages B253.

11. Suess, P.; Spiegel, L., Hold-up of mellapak structured packings. Chemical Engineering and Processing 1992, 31 (2), 119-124.

12. Rocha, J. A.; Bravo, J. L.; Fair, J. R., Distillation columns containing structured packings: a comprehensive model for their performance. 1. Hydraulic models. Industrial \& Engineering Chemistry Research 1993, 32 (4), 641-651.

13. Stichlmair, J.; Bravo, J. L.; Fair, J. R., General model for prediction of pressure drop and capacity of countercurrent gas/liquid packed columns. Gas Separation \&amp; Purification 1989, 3 (1), 19-28.

14. Valenz, L.; Rejl, F. J.; Linek, V., Gas and Liquid Axial Mixing in the Column Packed with Mellapak 250Y, Pall Rings 25, and Intalox Saddles 25 under Flow Conditions Prevailing in Distillation Columns. Industrial \& Engineering Chemistry Research 2010, 49 (20), 10016-10025.

15. Billet, R.; Schultes, M., Prediction of Mass Transfer Columns with Dumped and Arranged Packings: Updated Summary of the Calculation Method of Billet and Schultes. Chemical Engineering Research and Design 1999, 77 (6), 498-504.

16. Maćkowiak, J., Determination of flooding gas velocity and liquid hold-up at flooding in packed columns for gas/liquid systems. Chemical Engineering \& Technology 1990, 13 (1), 184-196.

17. Lockett, M. J., FLOODING OF ROTATING STRUCTURED PACKING AND ITS APPLICATION TO CONVENTIONAL PACKED-COLUMNS. Chem. Eng. Res. Des. 1995, 73 (A4), 379-384. 
18. Kuźniewska-lach, I., Estimation of phase velocities at flooding point in packed columns for any gas/liquid system. The Canadian Journal of Chemical Engineering 1999, 77 (3), 439-446. 